\title{
Multiomics Combined Analysis of Low-Phosphorus and Drought Stresses in Lotus Japonicus
}

\section{Ma Peijie+}

Grassland Research Institute of Guizhou Academy of Agricultural Sciences

\section{Li Yajiao+}

Grassland Research Institute of Guizhou Academy of Agricultural Sciences

\section{Shu Jianhong}

Grassland Research Institute of Guizhou Academy of Agricultural Sciences

\section{Wang Ziyuan}

Grassland Research Institute of Guizhou Academy of Agricultural Sciences

Chen Xi

Grassland Research Institute of Guizhou Academy of Agricultural Sciences

\section{Wang Xiaoli ( $\sim 398189177 @ q q . c o m$ )}

Grassland Research Institute of Guizhou Academy of Agricultural Sciences

\section{Research Article}

Keywords: Lotus japonicus, low phosphorus, drought stress, transcriptome, metabolome, proteome

Posted Date: October 5th, 2021

DOI: https://doi.org/10.21203/rs.3.rs-942639/v1

License: (c) (i) This work is licensed under a Creative Commons Attribution 4.0 International License.

Read Full License 


\section{Abstract}

\section{Background}

Lotus japonicus is a perennial herb in Leguminosae. It is a good feed source and improves soil. It is also an excellent honey source and medicinal plant. Low-phosphorus and drought stresses are among the main abiotic stress factors limiting the production of pulse roots.

\section{Methods}

In this experiment, the effects of low-phosphorus and drought stresses on Baimai roots were analyzed under three treatments: control (z|1), low-phosphorus stress (z|2) and drought stress (z|3).

\section{Results}

A total of 2176,3026 and 2980 differentially expressed genes were screened in zl1 vs. zl2, zl1 vs. zl3 and z|2 vs. z|3, respectively. The differentially expressed genes were enriched in functions related to cells, membranes, ion binding, enzyme activity and resistance to low-phosphorus and drought stresses. The enriched KEGG pathways included the MAPK signaling pathway-plant, flavor biosynthesis, starch and sucrose metabolism and plant hormone signal transmission. In particular, a large number of differentially expressed genes were enriched in the response to plant hormone signal transmission pathways among different treatments, and gene expression changes were analyzed. In addition, the differentially expressed genes identified under drought stress and the phase response genes identified under osmotic stress were upregulated. Differential metabolites were mainly enriched in the important metabolic pathways of flavonoid biosynthesis, arginine and proline metabolism and starch and sucrose metabolism.

Differentially expressed proteins were mainly enriched in $\mathrm{GO}$ terms related to cell, membrane, ion binding and enzyme activity functions, and the main enriched KEGG pathways included the ribosome, starch and sucrose metabolism and plant hormone signal transmission pathways.

\section{Conclusion}

In conclusion, these results of transcriptome, metabolome and proteome sequencing are helpful for understanding the response mechanisms, gene changes, metabolite changes and protein changes in Baimai roots under low-phosphorus and drought stress conditions to lay a foundation for future research on Lotus japonicus.

\section{Introduction}

- In the processes of growth and development, plants often suffer from a variety of biological and abiotic stresses, which affect the normal growth and development of plants and even cause death [1]. Drought stress is one of the main stresses affecting plant growth and development worldwide. The improvement of plant drought tolerance has become a research hotspot [2]. Phosphorus deficiency is another of the main problems affecting plant growth and development worldwide. 
Phosphorus is one of the large number of nutrients necessary for plant growth and development and plays a key role in the regulation of energy metabolism and nucleic acid and cell membrane synthesis [3]. In the production of economic plants in particular, drought stress and phosphorus deficiency seriously affect the growth, development and yield of plants, resulting in significant economic losses [4].

- Drought stress leads to the production of reactive oxygen species (ROS) in plants. Excessive ROS inhibit plant growth and even lead to cell death [5]. Key enzymes involved in the clearance of reactive oxygen species in plants, including superoxide dismutase (SOD), catalase (CAT), peroxidase (POD), ascorbic acid peroxidase (APX), glutathione reductase (GR) and catalase (POD), show changes under stress conditions[6]. The response of plants to drought stress is a complex biological process involving physiological, biochemical and molecular changes [7]. In this process, the expression of a large number of genes changes; these genes mainly belong to two categories. The first category comprises genes encoding "effector proteins" that directly protect plants from abiotic stress, including membrane protein genes, genes involved in the biosynthesis of various osmotic protective agents, photosynthesis-related genes, genes related to growth and development, chaperone-encoding genes, and genes encoding detoxification enzymes [8]. The second class of genes encode "regulatory proteins" that regulate downstream target gene expression related to stress responses, including genes encoding protein kinases (PKs), such as mitogen-activated PKs, ribosomal PKs and receptor PKs1, and transcription factors (TFs), such as AP2/ERF, AREB/ABF, bZIP, DREB, MYC/MYB and DREB.

- Phosphorus $(P)$ is necessary for normal plant growth and development, including playing roles in regulating energy metabolism, enzyme reactions and signal transduction [14]. To reduce the adverse effects of phosphorus stress, plants have evolved a variety of strategies, including repatterning root morphology to improve the absorption capacity, increasing the production and secretion of organic acids and phosphatases, establishing symbiotic relationships with arbuscular mycorrhizal fungi, and bypassing metabolic steps requiring ATP [15-18]. Variable phosphorus utilization rates depend on gene expression changes. Some key regulators modulate the plant response to phosphorus, including the MYB transcription factor Phr1, which can function as the central regulator of downstream genes [19]; WRKY and pho family members [20-21]; the miRNAs mirna399 and mirna827 [22]; the E3 ligases NLA and SIZ1 [23]; and IPS1/at4 [24]. Zmptf1 can improve the lowphosphorus tolerance of maize by regulating carbon metabolism and root growth [25].

- Transcriptomic analysis is an efficient way to study genome functions and related important pathways [26]. Metabonomics allows the detection and screening of metabolites with important biological significance and statistically significant differences in organisms and can therefore clarify the metabolic processes and change mechanisms of organisms [27]. Proteomics is a new research field that analyzes the dynamic changes in the protein composition, expression levels and modification states in cells from an overall perspective, thereby contributing to the understanding of interactions and relationships between proteins and revealing the laws of protein functions and cell biological activities [28]. Many studies have used transcriptomics, metabolomics and proteomics to 
study changes in genes, functions, metabolites and proteins in plants under stress [29-30]. At present, there is no available research on low-phosphorus and drought stresses. Therefore, we used transcriptomics, metabolomics and proteomics to explore the gene changes, functional changes, material changes and protein changes in Lotus japonicus under low-phosphorus and drought stresses to lay a foundation for the further targeted selection of low-phosphorus- and droughttolerance genes.

\section{Materials And Methods}

\subsection{Materials, handling and sampling}

Lotus japonicus was planted in the artificial climate room of the Guizhou Prataculture Research Institute. The seeds were sown in $11^{\star} 15 \mathrm{~cm}$ pots before planting, and the leaves were transplanted into bottles containing Hoagland nutrient solution with distilled water when 2-3 leaves had grown. The applied treatments were as follows: blank control (zl1) : 1/8-Hoagland nutrient solution, $\mathrm{pH} 8.1 \pm 0.5$, with $10 \mathrm{mM}$ $\mathrm{NaHCO} 3$ and $0.25 \mathrm{mM} \mathrm{NH} 4 \mathrm{H} 2 \mathrm{PO} 4$; low phosphorus (zI2): 1/8-Hoagland nutrient solution, $\mathrm{pH} 8.1 \pm 0.5$, with $10 \mathrm{mM} \mathrm{NaHCO3}$; and 20\% drought stress (zl3): Hogland nutrient solution, pH $8.1 \pm 0.5$, with $10 \mathrm{mM}$ $\mathrm{NaHCO}, 0.25 \mathrm{mM} \mathrm{NH} 4 \mathrm{H} 2 \mathrm{PO} 4$, and $200 \mathrm{~g} / \mathrm{L}$ polyethyleneglycol (PEG-6000). Five seedlings were planted in each bottle, and the nutrient solution was changed every 3 days. The plants were harvested after one month of culture. Indicators were assessed, and samples were collected.

\subsection{Transcriptome sequencing and quality control}

Advanced molecular biology equipment was used to detect the purity, concentration and integrity of RNA samples to ensure the use of qualified samples for transcriptome sequencing. After the samples were qualified, a library was constructed: (1) eukaryotic mRNA was enriched with oligo (DT) magnetic beads; (2) mRNA was randomly interrupted by adding fragmentation buffer; (3) the first cDNA strand was synthesized with six-base random primers (random hexamers) using mRNA as a template, after which the second cDNA strand was synthesized by adding buffer, dNTPs, RNase $\mathrm{H}$ and DNA polymerase $\mathrm{l}$, and the CDNA was purified with AMPure XP beads; (4) the purified double-stranded CDNA was subjected to terminal repair, a tail was added and sequenced, and fragment size selection was performed with AMPure XP beads; (5) finally, the cDNA library was enriched by PCR. After the construction of the library, the effective concentration of the library (library effective concentration $>2 \mathrm{~nm}$ ) was accurately quantified by Q-PCR to ensure its quality. After passing the library inspection, different libraries were pooled according to the target amount of offline data and sequenced on the Illumina platform. The quality of the obtained data was strictly controlled, and the following filtering methods were applied: (1) removal of reads containing linkers; and (2) removal of low-quality reads (including reads with an $\mathrm{N}$ percentage greater than $10 \%$, a quality value (Q) $\leq 10$ or more than $50 \%$ alkali bases in the whole read). The high-quality clean data obtained after the above series of quality control steps were provided in FASTQ format.

2.3 Transcriptome data and bioinformatic analysis 
The obtained clean data were compared with the reference genome of pulse roots by using HISAT2 [31]. The obtained mapped data were used for library quality evaluation, including insertion fragment length tests and randomness tests, and structure-level analyses such as alternative splicing analysis, new gene discovery and gene structure optimization were carried out [32]. Then, the StringTie maximum flow algorithm was to measure the levels of transcript or gene expression based on the index of fragments per kilobase of transcript per million fragments mapped (FPKM) values [33]. Differential expression analysis and the functional annotation and functional enrichment of differentially expressed genes were performed according to the expression levels of genes in different samples or different sample groups.

\subsection{Metabolome sample processing and data analysis}

To better understand the changes in different metabolites in the pulse roots under low-phosphorus stress and drought stress, three groups were selected for metabolite determination: the untreated control (zl1), low-phosphorus (z|2) and drought stress (z|3) groups; 6 samples were analyzed in each group, for a total of 18 samples. Metabolite extraction was performed according to the following step: weigh $50 \mathrm{mg}$ of sample, add $1000 \mu \mathrm{L}$ of an extract containing an internal standard (1000:2) (methanol;acetonitrile:water volumetric ratio $=2: 2: 1$, internal standard concentration $2 \mathrm{mg} / \mathrm{L}$ ), and perform vortex mixing for $30 \mathrm{~s}$; add ceramic beads, grind at $45 \mathrm{~Hz}$ for $10 \mathrm{~min}$, and perform ultrasonication for $10 \mathrm{~min}$ (ice water bath); leave standing at $-20^{\circ} \mathrm{C}$ for one hour; centrifuge at $12,000 \mathrm{rpm}$ at $4^{\circ} \mathrm{C}$ for $15 \mathrm{~min}$; carefully remove $500 \mu \mathrm{L}$ of the supernatant in an EP pipe; dry the extract in a vacuum concentrator; add $160 \mu \mathrm{L}$ of extract (acetonitrile:water volumetric ratio $=1: 1$ ) to redissolve the dried metabolites; vortex for $30 \mathrm{~s}$; perform ultrasonication in an ice water bath for $10 \mathrm{~min}$; centrifuge the sample at $12,000 \mathrm{rpm}$ at $4^{\circ} \mathrm{C}$ for $15 \mathrm{~min}$; carefully remove $120 \mu \mathrm{L}$ of the supernatant in a $2 \mathrm{ml}$ injection bottle; and collect $10 \mu \mathrm{L}$ of each sample mixed with the QC samples for analysis in a liquid chromatography-mass spectrometry (LC-MS) system. The LC-MS system used for metabolomic analysis was composed of a Waltz acquisition I-class plus ultrahigh-performance liquid chromatography (UHPLC) apparatus in series with a Waltz Xevo g2-xs QTOF high-resolution mass spectrometer. The chromatographic column was an acquisition UPLC HSS T3 chromatographic column $(1.8 \mu \mathrm{m} 2.1 * 100 \mathrm{~mm})$ purchased from Waltz. The original data collected by Masslynx v4.2 were used for peak extraction, peak alignment and other data processing operations with Genesis Qi software. Identification was conducted based on the online METLIN database and the BmK self-built database of the Genesis Qi software. At the same time, the theoretical fragments were identified. The mass number deviation was within 100 ppm [34].

\subsection{Proteome sample processing}

To better understand the changes in pulse root proteins under low-phosphorus stress and drought stress, three groups were selected for metabolite determination: the untreated control (zl1), low-phosphorus (zl2) and drought stress (zl3) groups; three samples were selected for protein determination in each group, for a total of 9 samples. Quality control of protein extraction was performed according to the following steps: add $300 \mu \mathrm{L}$ of $8 \mathrm{~m}$ urea (lysate: protease inhibitor 50:1); perform ultrasonication for $1 \mathrm{~s}$ followed by a $2 \mathrm{~s}$ hold for a total of $120 \mathrm{~s}$; centrifuge at $4^{\circ} \mathrm{C} 14,000 \mathrm{~g}$ for $20 \mathrm{~min}$; and perform protein quantification in the 
supernatant and SDS-PAGE quality control. Enzymatic desalting was conducted as follows: remove 100 $\mu \mathrm{g}$ of protein; add DTT at a final concentration of $10 \mathrm{~mm}$; incubate at $37^{\circ} \mathrm{C}$ for $1 \mathrm{~h}$; and restore to room temperature; add lam at a final concentration of $40 \mathrm{~mm}$; incubate away from light at room temperature for $45 \mathrm{~min}$; dilute the sample with ammonium bicarbonate to $\mathrm{pH}=8$; add trypsin at a protein-to-trypsin ratio $50: 1$; incubate overnight at $37^{\circ} \mathrm{C}$; the next day, add $50 \mu \mathrm{L} 0.1 \% \mathrm{FA}$ to terminate the reaction; desalt the sample with a C18 desalting column; activate the desalting column with $100 \%$ can; balance the column with $0.1 \% \mathrm{FA}$; load the sample onto the column; wash the column with $0.1 \% \mathrm{FA}$ to remove impurities; finally, perform elution with $70 \% \mathrm{ACN}$, collect the flow through solution, and perform freezedrying. Marking ot the samples was performed as follows: remove the TMT reagent; thaw it at room temperature; open the cover, and add $41 \mu \mathrm{L}$ of acetonitrile; shake for $5 \mathrm{~min}$ and centrifuge the mixture; add $100 \mu \mathrm{G}$ of TMT reagent to the enzyme-digested sample, and allow the reaction to occur at room temperature for $1 \mathrm{~h}$; add ammonia to terminate the reaction; mix, vortex oscillate and centrifuge the labeled samples; dry the samples by centrifugal vacuum freezing; mix the labeled samples with $100 \mu \mathrm{L}$ of mobile phase a to redissolve them; centrifuge at $14,000 \mathrm{~g}$ for $20 \mathrm{~min}$; collect the supernatant; and analyze the samples by HPLC at a flow rate of $0.7 \mathrm{~mL} / \mathrm{min}$ for classification.

\subsection{Proteome LC-MS/MS}

Proteome LC-MS/MS was performed as follows: prepare mobile phase liquid A ( $100 \%$ water; $0.1 \%$ formic acid) and liquid B ( $80 \%$ acetonitrile; $0.1 \%$ formic acid); dissolve the lyophilized powder with $10 \mu \mathrm{L}$ of solution; centrifuge at $14,000 \mathrm{~g}$ at $4{ }^{\circ} \mathrm{C}$ for $20 \mathrm{~min}$; collect $1 \mu \mathrm{g}$ of the supernatant for sample injection; and test the liquid quality. A Q Exactive HF-X mass spectrometer with a Nanospray Flex ${ }^{\mathrm{TM}}$ (NSI) ion source were used. The ion spray voltage was $2.4 \mathrm{kV}$; the ion transmission tube temperature was 275 degrees C; MS was conducted in data-dependent acquisition mode; the full scan range of MS was $\mathrm{m} / \mathrm{z}$ 407-1500; the first-level mass spectrometer resolution was 60,000 (200 m/z); AGC was $3 \times 106$; and the maximum Ctrap injection time was $20 \mathrm{~ms}$. The parent ion with an ion strength in the top 40 in the full scan was selected and disintegrated by high-energy collision cracking (HCD) method for secondary MS detection. The resolution of secondary MS was set to 45,000 (200 m/z); AGC was 5×104; the maximum injection time was $86 \mathrm{~ms}$; and the peptide fragmentation collision energy was set to $32 \%$ to generate the original MS data.

\section{Results}

\subsection{Appearance characteristics of Lotus japonicus under low-phosphorus and drought stress}

- Lotus japonicus was grown for one month under low-phosphorus and drought stresses. Compared with the control, the growth of the aboveground parts of the pulse roots became weaker, the stem became shorter and the number of leaves decreased (Fig. 1a, 1b). The growth potential of the underground root system of Baimai roots became weaker, the main roots became shorter, and the number of lateral roots decreased significantly (Fig. 1C, 1D). 


\subsection{Overview of transcriptome sequencing}

- Through the transcriptome analysis of 9 Lotus japonicus samples subjected to the control, lowphosphorus stress and drought stress treatments, a total of $57.09 \mathrm{~GB}$ clean data were obtained, the amount of clean data from each sample reached $6.05 \mathrm{~GB}$, and the q30 base percentage was $94.67 \%$ or more (Supplementary Table 1). In addition, we mapped the sequence base content and sequencing quality values to determine the sequencing quality (Supplementary Fig. 1). The clean reads of each sample were sequenced to obtain the genomes of pulse roots, and the alignment efficiency ranged from 82.44-84.02\% (Supplementary Table 2). Moreover, the coverage depth distribution map of the reads of each sample mapped against the reference genome (Supplementary

Fig. 2) and the distribution map of the reads of each sample mapped against different regions of the genome (Supplementary Fig. 3) were generated. The degree of random mRNA fragmentation was tested by simulating the results of mRNA fragmentation by assessing the distribution of mapped reads in each mRNA transcript (Supplementary Fig. 4). To test the effect of magnetic bead purification during library preparation, an insert fragment length test was performed (Supplementary Fig. 5). The number of genes sequenced from each sample was determined based on saturation, and the performance trend was the same (Supplementary Fig. 6). The gene expression distribution intervals in the transcriptomes of the 9 pulse root samples were highly conserved (Fig. 2A). The assessment of correlations (squared Pearson correlation coefficient) of expression between different samples showed that the correlations among the control, drought and low-phosphorus treatments were strong (Fig. 2B). Principal component analysis (PCA) showed that the gene expression patterns differed greatly under the control, drought and low-phosphorus treatments, indicating that the sample treatment results were reliable (Fig. 2C).

\subsection{Differentially expressed genes and enrichment analysis}

- Differentially expressed genes were determined by pairwise analyses between the control, lowphosphorus, and drought treatments using the DESeq2 tool (Supplement: Fig. 7). A total of 2176 differentially expressed genes were screened in zl1 vs. zl2(zl12), including 994 upregulated genes and 1182 downregulated genes (Supplement: Table 3). The screening of zl1 vs. zl3 (zl13) revealed 3026 differentially expressed genes, including 1990 upregulated genes and 1036 downregulated genes (Supplement: Table 4). A total of 2980 differentially expressed genes were screened in zl2 vs. z|3(z|23), among which 2115 were upregulated, and 865 were downregulated (Supplement: Table 5). In addition, Venn analysis of the three groups of differentially expressed genes was performed (Fig. 3A), and the numbers of common differentially expressed genes among different groups were recorded. zl12 and zl13 shared 700 differentially expressed genes, zl12 and zl23 shared 593 differentially expressed genes, zl13 and zl23 shared 1121 differentially expressed genes, and z|12, zl13 and zl23 shared 168 differentially expressed genes (Fig. 3A).

- To explore the function of differentially expressed genes between different treatments, we annotated the GO functions of differentially expressed genes between different treatments and displayed the 
top $20 \mathrm{GO}$ functions with significant enrichment (Fig. 3B). According to the differential expression gene enrichment functions of control zl1 vs. zl2(zl12), control zl1 vs. zl3(z|13) and control zl2 vs. zl3 (z|23), the genes identified in the three comparisons were significantly enriched in cell, membrane and plant type functions in the cellular component category. In addition, three kinds of photosystem functions were enriched under low-phosphorus-drought. In terms of molecular functions, the genes identified among the three comparison groups were significantly enriched in various enzyme activities and ion binding functions. In addition, the low-phosphorus control was enriched in transcription factor functions. Among biological processes, the genes of the three groups were mainly concentrated in various metabolic and transport-related functions. In addition, the GO enrichment results of the common differentially expressed genes of zl12, zl13 and zl23 were all in accord with the above results (Supplement, Fig. 8).

- To understand the KEGG pathway enrichment of differentially expressed genes, we focused on the KEGG pathway annotation of differentially expressed genes among the low-phosphorus control, drought control and low-phosphorus-drought treatments and identified the top 20 pathways with significant enrichment (Fig. 3C). The differentially expressed genes in zl12 were enriched in the plant hormone signal transduction, oxidative phosphorylation, peroxisome, and phosphate management pathways. The differentially expressed genes in z|13 were enriched in plant hormone signal transduction, MAPK signaling pathway-plant and flavonoid biosynthesis, which are pathways that regulate drought stress in plants. The differentially expressed genes in zl23 were enriched in the phosphatidylinositol signaling system and flavonoid biosynthesis, which regulate phosphoric acid and drought stress pathways. In addition, the differentially expressed genes common of zl12, zl13 and zl 23 were enriched in MAPK signaling pathway-plant and plant hormone signal transduction (Supplement, Fig. 8).

\subsection{Osmotic stress}

- The osmotic stress response is the core of the plant response to drought stress. Therefore, we mapped the differentially expressed genes identified in drought-related controls to osmotic stress regulatory networks (Supplement, Table 6). The results showed a total of 6 differentially expressed genes in the ABA signaling pathway, 2 of which were related to ABA receptor PYR/PYLs, and their expression levels were increased. One differentially expressed gene corresponded to RALF, and two differentially expressed genes corresponded to fer. One differentially expressed gene corresponded to GEF, and its expression was reduced. A total of 9 differentially expressed genes were included in the osmotic stress signaling pathway, 6 of which encoded MAPKs, and their expression was increased. The expression of three differentially expressed genes corresponding to RBOHB/C was increased. In addition, 72 differentially expressed genes encoded transcription factors. Most of the 22 WRKY genes were highly expressed under drought stress, and some of these genes were also highly expressed under low-phosphorus stress. The expression of 7 of $10 \mathrm{bhlhs}$ was increased. The expression of 10 of the 11 NACs was increased. The expression of 18 of the 24 mybs was increased. The expression of 2 of the 5 AP2s was increased. It is worth noting that POD and HKT, related to 
nonenzymatic antioxidants, were also ranked as ninth and first, respectively. The expression of POD increased and that of HKT decreased (Fig. 4).

\section{Note}

ABA, abscisic acid; PYR/PYLs, pyrabactin resistance 1-like protein; PP2C, type $2 \mathrm{C}$ protein phosphatases; SnRKs, Snf1 (sucrose nonfermenting-1)-related protein kinases; RBOHD/F, respiratory burst oxidase homolog D; SLAC1, slow anion channel 1; CPK/CBL, calcium-regulated phosphorylation systems; CAT, catalase; RALF, rapid alkalinization factor; FER, Feronia; GEF, guanine nucleotide exchange factor; MAPK, mitogen-activated protein kinase; ABFs, ABF, transcription factor; WRKY, WRKY transcription factor; bHIH, bHIH transcription factor; NAC, NAC transcription factor; MYB, MYB transcription factor; AP2, AP2/ERF transcription factor, GRAS, GRAS transcription factor; POD, peroxidase; GST, glutathione S-transferase zeta; HKTs, cation transporters.

\subsection{Plant hormone signal transduction pathway response gene analysis}

- We selected the FPKM values of differentially expressed genes enriched in the plant hormone signal transduction pathway from the zl1 vs. zl2(zl12), zl1 vs. zl3(zl13) and zl2 vs. zl3(zl23) comparisons for analysis. The results showed that among the zl12 differentially expressed genes, the auxin pathway was enriched in two gene types, and two $\mathrm{GH} 3$ genes and one SAUR gene were upregulated. The gibberellin pathway was enriched in 4 TF genes, and 3 of these genes were upregulated. Abscisic acid was enriched in two PYR/PYL genes, and their expression was upregulated. Only one $E R F 1 / 2$ gene was enriched in the ethylene pathway, and its expression was downregulated. Nine $J A Z$ genes were enriched in the jasmonic acid pathway, and their expression was upregulated. The salicylic acid pathway was enriched in 2 NPR1 genes, 3 TGA genes and 3 PR-1 genes, all of which were downregulated (Fig. 5A). Among the zl13 differentially expressed genes, the auxin pathway was enriched in one TIR1 gene, whose expression was downregulated; one $A U X / I A A$ gene, which was upregulated; six $\mathrm{GH} 3$ genes, 4 of which were upregulated; and three SAUR genes, one of which was upregulated. One AHP and one B-ARR gene were upregulated in the cytokinin pathway. The gibberellin pathway was enriched in two TF genes, one of which was upregulated. The abscisic acid pathway was enriched in two PYR/PYL genes, whose expression was upregulated. In the ethylene pathway, two EBF1/2 and two ERF1/2 genes were enriched and upregulated. The jasmonic acid pathway was enriched in $10 \mathrm{JAZ}$ genes, and their expression was upregulated. Two TGA genes were annotated in the salicylic acid pathway, and their expression was downregulated. Two PR-1 genes were also identified, one of which was upregulated (Fig. 5B). Among the zl23 differentially expressed genes, three $A U X / I A A$ genes were enriched in the auxin pathway, and one of the $A U X / I A A$ genes was upregulated. Five $G H 3$ genes were identified, four of which were upregulated. Three AHP genes were enriched in the cytokinin pathway, and two were upregulated. The gibberellin pathway was enriched in one TF gene, which was downregulated. The ABF gene was enriched in the abscisic acid pathway and was upregulated. The ethylene pathway enriched in two ERF1/2 genes, and their expression was 
upregulated. The jasmonic acid pathway was enriched in $6 \mathrm{JAZ}$ genes, and their expression was upregulated. The salicylic acid pathway was annotated with 2 PR-1 genes, both of which were upregulated (Fig. 5C).

\subsection{Transcription factors}

- We selected bZIP(67), PP2C (91), LEA (70), PLATZ (14) and ACX (3) transcription factors related to growth and development and stress and selected SPS (8), ALA (20) and PIP5K (17) gene families related to the phosphorus response. The measurement of gene expression differences showed that the $b Z I P$ and $P L A T Z$ families presented little difference in the number of members with significant expression under control, low-phosphorus and drought conditions. Most PP2C and $\angle E A$ genes were significantly expressed under drought conditions. Two ACX2 genes were significantly expressed under drought treatment. Most SPS genes were significantly expressed under low-phosphorus conditions. Most ALA genes were significantly expressed under drought conditions. Most PIP5K genes were significantly expressed under control conditions (Fig. 6).

\section{7 qRT - PCR testing of differentially expressed genes}

- To verify the reliability of the gene expression data obtained by transcriptome sequencing, 10 genes were randomly selected from 168 differentially expressed genes in the control group, low-phosphorus group and drought group for qRT-PCR verification. The qRT-PCR expression levels and expression trends of the differentially expressed genes were consistent with the transcriptome sequencing results, indicating high reliability of the transcriptional data (Fig. 7).

\subsection{Differential metabolite analysis}

- We analyzed 730 metabolites from 18 samples with 6 replicates under control, low-phosphorus and drought stresses(Fig. 8A). There were 390 different metabolites identified in the control group, among which 76 were upregulated, and 314 were downregulated. There were 397 differential metabolites identified in the drought control, among which 130 were upregulated, and 267 were downregulated. There were 332 different metabolites identified under low-phosphorus-drought stress, among which 209 were upregulated, and 123 were downregulated. A total of 620 differential metabolites were obtained (Supplement, Table 7), which were enriched in 59 KEGG pathways, including 55 metabolic-type pathways, and 41 metabolites were enriched in metabolic pathways. The biosynthesis of secondary metabolites was enriched in 26 kinds of metabolites (Fig. 8B). Among the top 20 pathways showing significant enrichment, the citrate cycle (TCA cycle), sucrose metabolism, arginine and proline metabolism and pentose phosphate pathway and other pathways were involved in plant responses to drought stress and phosphorylation (Fig. 8C). Notably, 74 kinds of common metabolites were screened, which were enriched in 18 KEGG pathways, including 17 metabolism-type pathways. Metabolic pathways and the biosynthesis of secondary metabolites were enriched in 7 kinds of metabolites (Fig. 8D). Furthermore, various flavonoid biosynthesis, 
arginine and proline metabolism, and glycerophospholipid metabolism pathways participate in the metabolic and phosphorylation reactions of substances under drought stress (Fig. 8E). In addition, heat map analysis of the quantitative results for 74 kinds of metabolites showed that 44 kinds of differential metabolites presented high contents under control conditions, 6 kinds of differential metabolites presented high contents under low-phosphorus conditions, and 24 kinds of differential metabolites presented high contents under drought stress (Fig. 8F).

\subsection{Differentially expressed protein analysis}

- A total of 470 protein-encoding genes were identified by the proteomic analysis of nine samples from the control, low-phosphorus and drought stress treatments (Supplement: Table 9). We analyzed the differentially expressed proteins among different treatments, and 71 differentially expressed proteins were identified in total (Fig. 9A), among which 7 differentially expressed proteins were found in the control group, including 3 upregulated and 4 downregulated proteins. There were 29 differentially expressed proteins related to drought control, including 16 upregulated and 13 downregulated proteins. There were 56 differentially expressed proteins identified under the low-phosphorusdrought treatment, 20 of which were upregulated and 36 of which were downregulated. In addition, 71 differentially expressed proteins were analyzed for enrichment. GO enrichment analysis showed that 41 differentially expressed proteins were related to metabolic processes, while 28 and 24 differentially expressed proteins were related to cellular processes and single organism processes, respectively (Fig. 9B). Neither immune system processes nor multiorganism processes were enriched in differentially expressed proteins. Among cellular components, the cell and cell part categories were enriched in 26 differentially expressed proteins, and the organelle category was enriched in 22 differentially expressed proteins. The membrane-enclosed lumen, nucleoid, virion and virion part categories were not enriched in differentially expressed proteins. Among molecular functions, binding and catalytic activities were enriched in 33 and 32 differentially expressed proteins, respectively. The antioxidant activity, molecular activity and protein tag categories were not enriched in differentially expressed proteins. The results of the KEGG pathway enrichment analysis showed that the ribosome pathway was enriched in 9 differentially expressed proteins, and the other 19 pathways all included 1 or 2 differentially expressed proteins. Sucrose metabolism and the phosphoric acid reaction pathway of carbon metabolism, starch and sucrose metabolism and pyruvate metabolism (Fig. 9C). In addition, heat maps were drawn based on the quantitative values of 71 differentially expressed proteins, 13 of which were high in the control group, and 22 of which were high in the low-phosphorus and drought groups. The quantitative values of most differentially expressed proteins under drought conditions were lower than those under the control and lowphosphorus treatments (Fig. 9D).

\section{Discussion}


- The stems and leaves of Lotus japonicus are soft, juicy and rich in carbohydrates. Lotus japonicus is a good feed source, improves the soil and is an excellent nectar source and medicinal plant [35]. However, there are no studies on low-phosphorus stress and drought stress in Lotus japonicus. Therefore, it is very important to improve the tolerance of Lotus japonicus to drought stress and lowphosphorus stress in production, and more studies are needed to explore the mechanism by which Lotus japonicuscopes with drought stress and low-phosphorus stress. In this study, the multiomics techniques of transcriptomics, metabolomics and proteomics were used to analyze the related functions, signaling pathways, differentially expressed genes, differentially expressed metabolites and differentially expressed proteins of Lotus japonicus under drought stress and low-phosphorus stress.

\subsection{Phenotypic changes in Lotus japonicus under drought and low-phosphorus stress}

- Plants suffer from drought stress, resulting in stunted plants, sparse and wilted branches and leaves, and in serious cases, plant death [36]. Under low-phosphorus stress, the taproot length and stem weight of Arabidopsis thaliana decrease significantly [37-38]. Under low-phosphorus stress, the stem dry matter and root dry matter contents of chickpea plants decrease to the same extent [39]. Low-phosphorus stress significantly reduces the biomass of wheat, especially the root biomass [40]. Lotus japonicus grown under normal environmental conditions had long stems and dense leaves, developed dense, deep roots, and grew vigorously. Under drought and low-phosphorus stress treatment, the shoots of the aboveground parts of Lotus japonicus became shorter, the number of leaves decreased, and the stems and leaves were sparse. In the underground parts, of the plant the number of roots decreased greatly, the roots became shorter, and the overall growth and development of the plant was reduced. These results indicated that drought and low-phosphorus stress severely hindered the growth and development of Lotus japonicus and seriously reduced the biomass of roots, stems and leaves.

\subsection{Differentially expressed genes and enrichment analysis}

- In this study, we screened differentially expressed genes between different treatments, and the results identified 2176 differentially expressed genes in the low-phosphorus control, 3026 differentially expressed genes in the drought control, and 2980 differentially expressed genes in the low-phosphorus treatment. According to the top 20 functions showing $\mathrm{GO}$ enrichment, the differentially expressed genes identified under the low-phosphorus control and drought control treatments were mainly enriched in cell, plant type and membrane functions. The differentially expressed genes identified under the low-phosphorus-drought treatment were mainly enriched in membrane and photosystem functions. Studies have shown that when plants are subjected to stress, the physiological and biochemical processes of cells and the functions of membrane systems in the body show corresponding changes, including the selective absorption of mineral ions for 
osmotic regulation and upregulation of antioxidants and osmoprotectants accumulated in the cytoplasm and organelles [41-42]. The accumulation of osmotic substances such as proline, betaine, and sugars maintains cell function by stabilizing cell osmosis, which in turn maintains the structure and function of enzymes by maintaining the cell water content and assisting in ROS clearance [43-44]. The results showed that after drought and low-phosphorus stress, the expression levels of genes related to the cell response and membrane function were changed, and the responses were related to resistance to the harm caused by low-phosphorus and drought stresses. Plant growth and functioning are closely related to phosphorus acquisition and the function of photosynthetic mechanisms [45]. Phosphorus is necessary for maintaining photoreactions, photophosphorylation and carbohydrate production [46]. The results indicated that the expression levels of responsive genes related to photosystems I and II of Lotus japonicus were changed under low-phosphorus stress, which promoted a series of photosynthetic processes. In terms of molecular functions, the differentially expressed genes identified under low-phosphorus stress were mainly enriched in various enzyme activities and ion binding functions, while the differentially expressed genes identified under low-phosphorus-drought were mainly enriched in various enzyme activities and ion binding functions. Plants adapt to short-term abiotic stress through physiological regulation [47]. Plant activity is increased by osmotic regulation or ROS removal. Proline and sugar play important roles in osmotic regulation, and SOD, POD and CAT mediate ROS clearance. In particular, differentially expressed genes related to oxidoreductase activity (oxidoreductase activity, oxygenase activity, peroxidase activity and oxidoreductase activity) (activity) functions were enriched under drought conditions, which improved the ROS clearance-related reactions of Lotus japonicus [48-50]. However, many differentially expressed genes were enriched in transcription factor function under low-phosphorus stress, indicating that multiple transcription factors were actively expressed to regulate the Lotus japonicus response to low-phosphorus stress. These results indicated that not only were various enzyme activities regulated to cope with stress in Lotus japonicus but that many other functions were also deployed to cope with drought and low-phosphorus stress. Among biological processes, genes that were differentially expressed in the low-phosphorus control, drought control and low-phosphorus-drought treatments were enriched in various metabolic and transport functions, especially in flavonoid metabolic processes. Flavonoids can improve the plant response to various kinds of stress [51]. We also analyzed the significant GO functions of differentially expressed gene shared between two treatments, and the results were similar. The analysis of the KEGG enrichment pathways of the differentially expressed genes between different treatments showed that multiple response pathways and metabolic pathways were associated with stress tolerance in the low-phosphorus control, drought control and low-phosphorus-drought treatments. These pathways included plant hormone signal transduction, MAPK signaling pathway-plant and flavonoid biosynthesis. These results indicated that Lotus japonicus deployed a variety of response mechanisms to resist the harm caused by adverse stress when subjected to low-phosphorus and drought stresses.

\subsection{Plant hormone signal transduction}


- We investigated the differentially expressed response genes of Lotus japonicus in the plant hormone signal transduction pathway under the low-phosphorus and drought controls and low-phosphorus stress. We focused on the hormone pathways showing enrichment by multiple genes. Genes in the auxin pathway regulate plant growth and cell development and improve the ability of plants to resist stress. In particular, GH3 regulates the growth and development of primary roots in Arabidopsis [52]. GH3 and SAUR genes were enriched in the roots of Lotus japonicus under low-phosphorus and drought stresses, and the expression activity of upregulated genes was enhanced under lowphosphorus and drought stresses, thus improving the resistance of Lotus japonicus to stress. In addition, the auxin pathway was enriched in one downregulated TIR1 gene and one upregulated AUX/IAA gene under drought stress. TIR1 is an important auxin receptor gene, and TIR1 and AUX/IAA play an important role in regulating plant root growth and development and resistance to drought stress [53-54]. These results indicated that more regulatory genes were involved in the auxin pathway in Lotus japonicus under drought stress. The cytokinin pathway plays an important role in regulating plant cell division and bud growth. Under low-phosphorus and drought stresses, four TF genes (PIF3 and PIF4) were enriched. PIF3 and PIF4 are essential photochrome interaction factors for photoinduced signal transduction in plants [55]. PIF4 also regulates plant growth and development and hypocotyl elongation in response to blue light under heat stress [56]. In the absence of phosphorus, chloroplasts and other phosphoric acid reactions were greatly reduced. Under low-phosphorus stress, the photosynthetic efficiency of Lotus japonicus decreased, which affected plant growth and development. Three upregulated genes (including PIF3 and PIF4) were enriched to enhance the response of Lotus japonicus to low-phosphorus stress. The ethylene pathway not only regulates fruit ripening but also regulates plant resistance to stress. Lotus japonicus showed the enrichment of one downregulated ERF1/2 gene under low-phosphorus stress and two EBF1/2 genes and two ERF1/2 genes under drought stress, all of which were upregulated. Related studies have shown that the eBF1/2 and ERF1/2 genes can regulate plant resistance to stress and seedling growth and development [57]. In addition, ERF1 regulates plant root growth [58]. Furthermore, the ethylene pathway plays an important role in the resistance to drought stress and root growth in Lotus japonicus under drought stress. The jasmonic acid pathway regulates plant signaling, resistance to stress, and plant growth and development. Under low-phosphorus and drought stresses, 9 and $10 J A Z$ genes were enriched and upregulated, respectively. The JAZ gene family has been extensively studied and shown to enable plants to resist pathogen infection and drought and saline-alkali stress and to play roles in signal transduction, among other functions [59]. These results suggest that the $J A Z$ gene family plays an important role in the low-phosphorus and drought stress responses of rhizomes. The salicylic acid pathway mainly improves plant resistance to disease. The NPR1, TGA and PR-1 genes were enriched and downregulated under low-phosphorus and drought stresses, indicating that Lotus japonicus could show reduced resistance to pathogens under phosphorus deficiency and drought. In particular, low-phosphorus stress may greatly reduce the resistance of rhizomes to pathogens. Finally, the number of differentially expressed response genes enriched in the plant hormone signal transduction pathway under low-phosphorus-drought stress was much lower, and most genes were highly expressed under drought. These results 
indicated that Lotus japonicus presented a stronger ability to resist drought stress than lowphosphorus stress.

\subsection{Osmotic stress response gene}

- Osmotic stress can be induced by drought and salt stress, and secondary messengers can be synthesized; the changes in secondary messengers can then be used as signals to activate different signal cascades. They are the main regulators of signal transduction and can effectively link external stimuli with cellular responses, thereby activating the expression of downstream functional genes [60]. In this study, we focused on the analysis of differentially expressed genes under drought stress and identified 72 differentially expressed genes related to osmotic stress signal transduction, including 2 PYR/PYL, 1 RALF, 2 FER and 6 MAPK genes. These results indicated that osmotic stressrelated genes were highly activated under Lotus japonicus stress. Other transcription factor-related genes were also upregulated, suggesting that Lotus japonicus has the ability to upregulate genes to resist drought stress. The GO enrichment analysis of genes that were differentially expressed under drought showed that the transporter activity, transmembrane transporter activity and cell membrane terms, which are closely related to osmotic stress, were significantly enriched under the two stresses imposed in this study. In addition, the differentially expressed genes were significantly enriched in redox enzyme activity, transmembrane transporter activity and transmembrane activity. Additionally, differentially expressed genes with the same expression patterns were also enriched in KEGG pathways such as amino acid metabolism, carbohydrate metabolism, signal transduction and cell process, and ion channel pathways. These findings are similar to those previously observed in Arabidopsis, alfalfa, rice, and red bean [61-63]. These results indicate that osmotic stress occurs in Lotus japonicus under drought stress. At this point, amino acid metabolism, antioxidant enzymes and other defense systems will be activated through ion channels and signal transduction systems to play a role in coping with stress.

\subsection{Differential metabolites and proteins analysis}

- Metabolites are a basis of organismal phenotypes, and their analysis can help to understand biological processes and their mechanisms more intuitively and effectively. Fifty-five KEGG pathways of 620 different metabolites were metabolism-type pathways. Sucrose metabolic pathways, including the citrate cycle (TCA cycle), starch and sucrose metabolism, arginine and proline metabolism and the pentose phosphate pathway, among others, are involved in the responses of Baimai roots to drought and low-phosphorus stresses. Notably, 74 common metabolites may be affected by both low-phosphorus and drought stresses, and KEGG pathways including flavonoid biosynthesis, arginine and proline metabolism and glycerophospholipid metabolism may regulate Lotus japonicus growth in response to low-phosphorus and drought stresses. In addition, the greatest number (74) of differential metabolites with high levels were found in the control group, followed by the drought stress group, while the low-phosphorus stress showed 
the fewest differential metabolites with high levels. The results indicated that the metabolite production of Lotus japonicus decreased greatly under low-phosphorus and drought stresses.

- Proteomics is a new research field involving the analysis of dynamic changes of the protein composition, expression levels and modification states in cells, understanding the interactions and connections between proteins, and revealing the laws of protein function and cell biological activities. We focused on the analysis of differentially expressed proteins in Lotus japonicus under control, low-phosphorus stress and drought stress treatments. The GO analysis of all 71 differentially expressed proteins showed that most of the differentially expressed proteins in the biological processes category were involved in cellular processes and metabolic processes, whereas most of the differentially expressed proteins in the cell component category were related to cell and membrane functions. Most of the differences in the molecular function category were related to binding and catalytic activity functions. The results indicated that the proteins related to the cell responses, membrane functions and enzyme activities of Lotus japonicus showed the greatest changes under low-phosphorus and drought stresses. The results of the KEGG pathway analysis showed that the ribosome was enriched in 9 differentially expressed proteins, indicating that many proteins in this pathway showed expression changes. Sucrose metabolism, the biosynthesis of amino acids and plant hormone signal transduction react to protein changes. The above analysis indicated that Lotus japonicus underwent changes in various functions and response pathways under low-phosphorus and drought stresses, which improved the resistance of Lotus japonicus.

\section{Conclusion}

In this study, transcriptomic, metabolomic and proteomic analyses were performed on Lotus japonicus under low-phosphorus and drought stresses. Differentially expressed genes, plant hormone signal transduction, osmotic stress processes, transcription factor expression, and changes in differentially expressed metabolites and differentially expressed proteins were analyzed. We found that many genes and important functional and metabolic pathways were involved in the regulation of Lotus japonicus under low-phosphorus and drought stresses. This in-depth research on the changes in genes, functions and pathways in Lotus japonicus under phosphorus deficiency and drought stresses lays a foundation for future research on important genes and substances related to phosphorus deficiency and drought stresses in Lotus japonicus.

\section{Declarations}

Ethics approval and consent to participate: The Lotus japonicus accessions materials used in the experiment were supplied by Pratacultural Research Institute of Guizhou Academy of Agricultural Sciences, China. These plant materials are widely used all over the world and no permits are required for the collection of plant samples. Experimental research and field studies on plants, including the collection of plant material, complied with relevant institutional, national, and international guidelines and legislation. Furthermore, this article did not contain any studies with human participants or animals and 
did not involve any endangered or protected species. All experiments and data analyses were conducted in Guizhou. All authors contributed to the study and approved the final version for submission. The manuscript has not been submitted to any other journal.

Consent for publication: YES.

Availability of data and materials: The materials were taken from the Grassland Research Institute of Guizhou Academy of Agricultural Sciences, and the data were entrusted to baimike company for analysis. Data owner】Wang Xiaoli. E-mail: 398189177@qq.com.

Competing interests: No competitive interest.

Author Contributions: Wang Xiaoli designed and managed the study. Ma Peijie, Li Yajiao drafted and revised the manuscript. Ma Peijie, Shu Jianhong performed the analysis. Jiahui Yang, Li Yajiao, Chen Xi, Wang Ziyuan participated in sample collection and conducted experiments. All authors read and approved the final manuscript.

Funding: This work was supported by Qkehe foundation [2019] No. 1449), Guizhou innovative talent team (qkehe platform talent [2020] 5005), Guizhou high-level innovative talent training (qkehe platform talent [2018] 5634), youth fund of Guizhou Academy of Agricultural Sciences (Youth Fund of Guizhou Academy of Agricultural Sciences [2019] No. 23).

\section{References}

1. Cejudo Francisco Javier,Sandalio Luisa M,Van Breusegem Frank. Understanding plant responses to stress conditions: redox-based strategies.[J]. Journal of experimental botany,2021,72(16):5785-5788.

2. Choukri Hasnae,Hejjaoui Kamal,El Baouchi Adil,El haddad Noureddine,Smouni Abdelaziz,Maalouf Fouad,Thavarajah Dil,Kumar Shiv. Heat and Drought Stress Impact on Phenology, Grain Yield, and Nutritional Quality of Lentil (Lens culinaris Medikus)[J]. Frontiers in Nutrition,2020:23;7:596307.

3. Krishnapriya Vengavasi,Renu Pandey. Root acidification, a rapid method of screening soybean genotypes for low-phosphorus stress[J]. Indian Journal of Genetics and Plant Breeding (The),2016,76(2):213-216.

4. Shahzad Aqeel,Ullah Sana,Dar Afzal Ahmed,Sardar Muhammad Fahad,Mehmood Tariq,Tufail Muhammad Aammar,Shakoor Awais,Haris Muhammad. Nexus on climate change: agriculture and possible solution to cope future climate change stresses.[J]. Environmental science and pollution research international,2021,28(12):1-22.

5. Reddy, A. R., Chaitanya, K. V., and Vivekanandan, M. (2004). Drought-induced responses of photosynthesis and antioxidant metabolism in higher plants. J. Plant Physiol. 161, 1189-1202.

6. Peisen Su,Jun Yan,Wen Li,Liang Wang,Jinxiao Zhao,Xin Ma,Anfei Li,Hongwei Wang,Lingrang Kong. A member of wheat class III peroxidase gene family, TaPRX-2A, enhanced the tolerance of salt stress[J]. BMC Plant Biology,2020,20(1):392. 
7. Osakabe, Y., Osakabe, K., Shinozaki, K., and Tran, L. S. Response of plants to water stress. Front. Plant Sci. 2014.5:86.

8. Ramalingam Abirami,Kudapa Himabindu,Pazhamala Lekha T.,Garg Vanika,Varshney Rajeev K. Gene Expression and Yeast Two-Hybrid Studies of 1R-MYB Transcription Factor Mediating Drought Stress Response in Chickpea (Cicer arietinum L.)[J]. Frontiers in Plant Science,2015.6:1117.

9. Xu, Y. et al. Transcriptome sequencing and whole genome expression profling of chrysanthemum under dehydration stress. Bmc

10. Genomics. 2013.14:1-15.

11. Wang Dan,Chen Qiyang,Chen Weiwei,Liu Xinya,Xia Yan,Guo Qigao,Jing Danlong,Liang Guolu. A WRKY Transcription Factor, EjWRKY17, from Eriobotrya japonica Enhances Drought Tolerance in Transgenic Arabidopsis.[J]. International journal of molecular sciences,2021,22(11):5593.

12. The bZIP Transcription Factor GmbZIP15 Negatively Regulates Salt- and Drought-Stress Responses in Soybean.[J]. International journal of molecular sciences,2020,21(20):1-19.

13. Wang Yujiao,Zhang Yong,Fan Chunjie,Wei Yongcheng,Meng Jingxiang,Li Zhen,Zhong Chonglu. Genome-wide analysis of MYB transcription factors and their responses to salt stress in Casuarina equisetifolia.[J]. BMC plant biology,2021,21(1):328.

14. Raghothama KG. Phosphate acquisition. Annu Rev Plant Physiol Mol Biol. 1999;50:665-93.

15. López-Arredondo DL, Leyva-González MA, González-Morales SI, López-BucioJ, Herrera-Estrella L. Phosphate nutrition: improving low-phosphate tolerance in crops. Annu Rev Plant Biol. 2014;65:95123.

16. López-Bucio J, Hernández-Abreu E, Sánchez-Calderón L, Nieto-Jacobo MF, Simpson J, HerreraEstrella L. Phosphate availability alters architecture and causes changes in hormone sensitivity in the Arabidopsis root system. Plant Physiol. 2002;129:244-56.

17. Bucher M. Functional biology of plant phosphate uptake at root and mycorrhiza interfaces. New Phytol. 2007;173:11-6.

18. Ganie AH, Ahmad A, Pandey R, Aref IM, Yousuf PY, Ahmad S, et al. Metabolite profiling of low-P tolerant and low-P sensitive maize genotypes under phosphorus starvation and restoration conditions. PLoS One. 2015;10:e0129520.

19. Rubio V, Linhares F, Solano R, Martín AC, Iglesias J, Leyva A, et al. A conserved MYB transcription factor involved in phosphate starvation signaling both in vascular plants and in unicellular algae. Genes Dev. 2001;15:2122-33.

20. Su T, Xu Q, Zhang FC, Chen Y, Li LQ, Wu WH, et al. WRKY42 modulates phosphate homeostasis through regulating phosphate translocation and acquisition in Arabidopsis. Plant Physiol. 2015;167:1579-91.

21. Wang H, Xu Q, Kong YH, Chen Y, Duan JY, Wu WH, et al. Arabidopsis WRKY45 transcription factor activates PHOSPHATE TRANSPORTER1;1 expression in response to phosphate starvation. Plant Physiol. 2014;164:2020-9. 
22. Kant S, Peng M, Rothstein SJ. Genetic regulation by NLA and microRNA827 for maintaining nitratedependent phosphate homeostasis in Arabidopsis. PLoS Genet. 2011;7:e1002021.

23. Miura K, Rus A, Sharkhuu A, Yokoi S, Karthikeyan AS, Raghothama KG, et al. The Arabidopsis SUMO E3 ligase SIZ1 controls phosphate deficiency responses. Proc Natl Acad Sci U S A. 2005;102:77605.

24. Hou $X$, Wu P, Jiao F, Jia Q, Chen $H$, Yu J, et al. Regulation of the expression of OsIPS1 and OsIPS2 in rice via systemic and local Pi signaling and hormones. Plant Cell Environ. 2005;28:353-64.

25. Li Z, Gao Q, Liu Y, He C, Zhang X, Zhang J. Overexpression of transcription factor ZmPTF1 improves low phosphate tolerance of maize by regulating carbon metabolism and root growth. Planta. 2011;233:1129-43.

26. Yongkun Chen,Canhui Li,Jing Yi,Yu Yang,Chunxia Lei,Ming Gong. Transcriptome Response to Drought, Rehydration and Re-Dehydration in Potato[J]. International Journal of Molecular Sciences,2019,21(1):159.

27. Zhangchi Ning,Cheng Lu,Yuxin Zhang,Siyu Zhao,Baoqin Liu,Xuegong Xu,Yuanyan Liu. Application of Plant Metabonomics in Quality Assessment forLarge-Scale Production of Traditional Chinese Medicine[J]. Planta Med,2013:897-908.

28. Yanmei Chen,Yi Wang,Jun Yang,Wenbin Zhou,Shaojun Dai.Exploring the diversity of plant proteome[J].Journal of Integrative Plant Biology,2021,63(07):1197-1210.

29. Chen Xiaoqiong,Tao Yu,Ali Asif,Zhuang Zhenhua,Guo Daiming,Guo Qiaoling,Riaz Asad,Zhang Hongyu,Xu Peizhou,Liao Yongxiang,Wang Jing,Sun Changhui,Xiang Quanju,Wu Xianjun. Transcriptome and Proteome Profiling of Different Colored Rice Reveals Physiological Dynamics Involved in the Flavonoid Pathway.[J]. International journal of molecular sciences,2019,20(10):2463.

30. Piasecka Anna,Kachlicki Piotr,Stobiecki Maciej. Analytical Methods for Detection of Plant Metabolomes Changes in Response to Biotic and Abiotic Stresses.[J]. International journal of molecular sciences,2019,20(2):379.

31. Kim D, Langmead B, Salzberg S L. HISAT: a fast spliced aligner with low memory requirements[J]. Nature methods, 2015, 12(4): 357-360.

32. McKenna A, Hanna M, Banks E, et al. The Genome Analysis Toolkit: a MapReduce framework for analyzing next-generation DNA sequencing data. Genome Research. 2010, 20(9): 1297-1303.

33. Pertea M, Pertea G M, Antonescu C M, et al. StringTie enables improved reconstruction of a transcriptome from RNA-seq reads[J]. Nature biotechnology, 2015, 33(3): 290-295.

34. William O. Slade,Emily G. Werth,Evan W. McConnell,Sophie Alvarez,Leslie M. Hicks. Quantifying Reversible Oxidation of Protein Thiols in Photosynthetic Organisms[J]. Journal of The American Society for Mass Spectrometry,2015,26(4):631-640.

35. Liu Jing,Liu Miao Xia,Qiu Li Ping,Xie Fang. SPIKE1 Activates the GTPase ROP6 to Guide the Polarized Growth of Infection Threads in Lotus japonicus[J]. The Plant Cell,2020,32(12): 3774-3791.

36. Bano Nasreen,Fakhrah Shafquat,Mohanty Chandra Sekhar,Bag Sumit Kumar. Genome-Wide Identification and Evolutionary Analysis of Gossypium Tubby-Like Protein (TLP) Gene Family and 
Expression Analyses During Salt and Drought Stress [J]. Frontiers in Plant Science,2021. 12:667929.

37. Reymond, M. et al. Identifcation of QTL controlling root growth response to phosphate starvation in Arabidopsis thaliana. Plant Cell \& Environment. 2010. 29, 115-125.

38. Narang, R. A., Bruene, A. \& Altmann, T. Analysis of phosphate acquisition efciency in diferent arabidopsis accessions. Plant Physiol. 2000. 124, 1786-1799.

39. Alloush, G. A. Responses of hydroponically-grown chickpea to low phosphorus: pH changes, nutrient uptake rates, and root morphological changes. Agronomie. 2003. 23, 123-133.

40. Jun Wang,Qin Qin,Jianjun Pan,Lijuan Sun,Yafei Sun,Yong Xue,Ke Song. Transcriptome analysis in roots and leaves of wheat seedlings in response to low-phosphorus stress[J]. Scientific Reports,2019,9:1-2.

41. Mantova Marylou,MenezesSilva Paulo E,Badel Eric,Cochard Hervé,TorresRuiz José M. The interplay of hydraulic failure and cell vitality explains tree capacity to recover from drought.[J]. Physiologia plantarum,2021,172(1):247-257.

42. Chen Keqin,Guo Yunna,Song Mengru,Liu Lifu,Xue Hao,Dai Hongyan,Zhang Zhihong. Dual role of MdSND1 in the biosynthesis of lignin and in signal transduction in response to salt and osmotic stress in apple.[J]. Horticulture research,2020,7(1):204.

43. Helga Koenigshofer,Hans-Georg Loeppert. The up-regulation of proline synthesis in the meristematic tissues of wheat seedlings upon short-term exposure to osmotic stress[J]. Journal of Plant Physiology,2019,237:21-29.

44. SAJJAD MOHARRAMNEJAD,OMID SOFALIAN,MOSTAFA VALIZADEH,ALI ASGARI,MOHAMMADREZA SHIRI. Proline, glycine betaine, total phenolics and pigment contents in response to osmotic stress in maize seedlings[J]. Journal of BioScience and Biotechnology,2015,4(3):313-319.

45. Thuynsma, R.; Kleinert, A.; Kossmann, J.; Valentine, A.J.; Hills, P.N. The effects of limiting phosphate on photosynthesis and growth of <bi>Lotus japonicus</bi>. S. Afr. J. Bot. 2016, 104, 244-248.

46. Li, H.; Yang, Y.; Zhang, H.; Chu, S.; Zhang, X.; Yin, D.; Yu, D.; Zhang, D. A genetic relationship between phosphorus efficiency and photosynthetic traits in soybean as revealed by QTL analysis using a high-density genetic map. Front. Plant Sci. 2016, 7.

47. Hafeez Muhammad Bilal,Zahra Noreen,Zahra Kiran,Raza Ali,Khan Aaliya,Shaukat Kanval,Khan Shahbaz. Brassinosteroids: Molecular and physiological responses in plant growth and abiotic stresses[J]. Plant Stress,2021,2:100029.

48. Lin Ze,Wang Yue-Li,Cheng Li-Sha,Zhou Li-Lang,Xu Qiu-Tao,Liu Dong-Cheng,Deng Xiang-Yi,Mei FangZhu,Zhou Zhu-Qing. Mutual regulation of ROS accumulation and cell autophagy in wheat roots under hypoxia stress[J]. Plant Physiology and Biochemistry,2021,158:91-102.

49. Khaled A. A. Abdelaal,Yasser S.A. Mazrou,Yaser M. Hafez. Silicon Foliar Application Mitigates Salt Stress in Sweet Pepper Plants by Enhancing Water Status, Photosynthesis, Antioxidant Enzyme Activity and Fruit Yield[J]. Plants,2020,9(6):733.

50. Lu-Lu Yu,Yang Liu,Cui-Jiao Liu,Feng Zhu,Zheng-Quan He,Fei Xu. Overexpressed $\beta$-cyanoalanine synthase functions with alternative oxidase to improve tobacco resistance to salt stress by 
alleviating oxidative damage[J]. FEBS Letters,2020,594(8):1284-1295.

51. Sina Siavash Moghaddam,Rusli Ibrahim,Christos A. Damalas,Seyyed Ali Noorhosseini. Effects of Gamma Stress and Carbon Dioxide on Eight Bioactive Flavonoids and Photosynthetic Efficiency in Centella asiatica[J]. Journal of Plant Growth Regulation,2017,36(4):957-969.

52. Sadaf Khan,Julie M. Stone. Arabidopsis thaliana GH3.9 influences primary root growth[J]. Planta,2007,226(1):21-34.

53. Ken-ichiro Hayashi,Xu Tan,Ning Zheng,Tatsuya Hatate,Yoshio Kimura,Stefan Kepinski,Hiroshi Nozaki. Small-Molecule Agonists and Antagonists of F-Box Protein-Substrate Interactions in Auxin Perception and Signaling[J]. Proceedings of the National Academy of Sciences of the United States of America,2008,105(14):5632-5637.

54. E. Liscum,J.W. Reed. Genetics of Aux/IAA and ARF action in plant growth and development[J]. Plant Molecular Biology,2002,49(3-4):387-400.

55. Min Ni,James M Tepperman,Peter H Quail. PIF3, a Phytochrome-Interacting Factor Necessary for Normal Photoinduced Signal Transduction, Is a Novel Basic Helix-Loop-Helix Protein[J]. Cell,1998,95(5):657-667.

56. Maria A. Koini,Liz Alvey,Trudie Allen,Ceinwen A. Tilley,Nicholas P. Harberd,Garry C. Whitelam,Keara A. Franklin. High Temperature-Mediated Adaptations in Plant Architecture Require the bHLH Transcription Factor PIF4[J]. Current Biology,2009,19(5):408-413.

57. Yang Huanhuan,Sun Yaoguang,Wang Hexuan,Zhao Tingting,Xu Xiangyang,Jiang Jingbin,Li Jingfu. Genome-wide identification and functional analysis of the ERF2 gene family in response to disease resistance against Stemphylium lycopersici in tomato.[J]. BMC plant biology,2021,21(1):72.

58. Mao JieLi,Miao Zi-Qing,Wang Zhen,Yu Lin-Hui,Cai Xiao-Teng,Xiang Cheng-Bin. Correction: Arabidopsis ERF1 Mediates Cross-Talk between Ethylene and Auxin Biosynthesis during Primary Root Elongation by Regulating ASA1 Expression.[J]. PLoS genetics,2016,12(5):e1006076.

59. Jiazhi Shen,Zhongwei Zou,Hongqing Xing,Yu Duan,Xujun Zhu,Yuanchun Ma,Yuhua Wang,Wanping Fang. Genome-Wide Analysis Reveals Stress and Hormone Responsive Patterns of JAZ Family Genes in Camellia Sinensis[J]. International Journal of Molecular Sciences,2020,21(7):2433.

60. Luo, D.; Zhou, Q.; Wu, Y.; Chai, X.; Liu, W.; Wang, Y.; Yang, Q.; Wang, Z.; Liu, Z. Full-length transcript sequencing and comparative transcriptomic analysis to evaluate the contribution of osmotic and ionic stress components towards salinity tolerance in the roots of cultivated alfalfa (Medicago sativa L.). BMC Plant Biol. 2019, 19.

61. Zhu, Z.; Chen, H.; Xie, K.; Liu, C.; Li, L.; Liu, L.; Han, X.; Jiao, C.; Wan, Z.; Sha, A. Characterization of drought-responsive transcriptome during seed germination in Adzuki bean (L.) by PacBio SMRT and Illumina Sequencing. Front. Genet. 2020, 11, 996.

62. Wong, C.E.; Li, Y.; Labbe, A.; Guevara, D.; Nuin, P.; Whitty, B.; Diaz, C.; Golding, G.B.; Gray, G.R.; Weretilnyk, E.A.; et al.Transcriptional profiling implicates novel interactions between abiotic stress and hormonal responses in Thellungiella, a close relative of Arabidopsis. Plant Physiol. 2006, 140, 1437-1450. 
63. Rabbani, M.A.; Maruyama, K.; Abe, H.; Khan, M.A.; Katsura, K.; Ito, Y.; Yoshiwara, K.; Seki, M.; Shinozaki, K.; YamaguchiShinozaki, K. Monitoring expression profiles of rice genes under cold, drought, and high-salinity stresses and abscisic acid application using cDNA microarray and RNA gel-blot analyses. Plant Physiol. 2003, 133, 1755-1767.

\section{Figures}

A

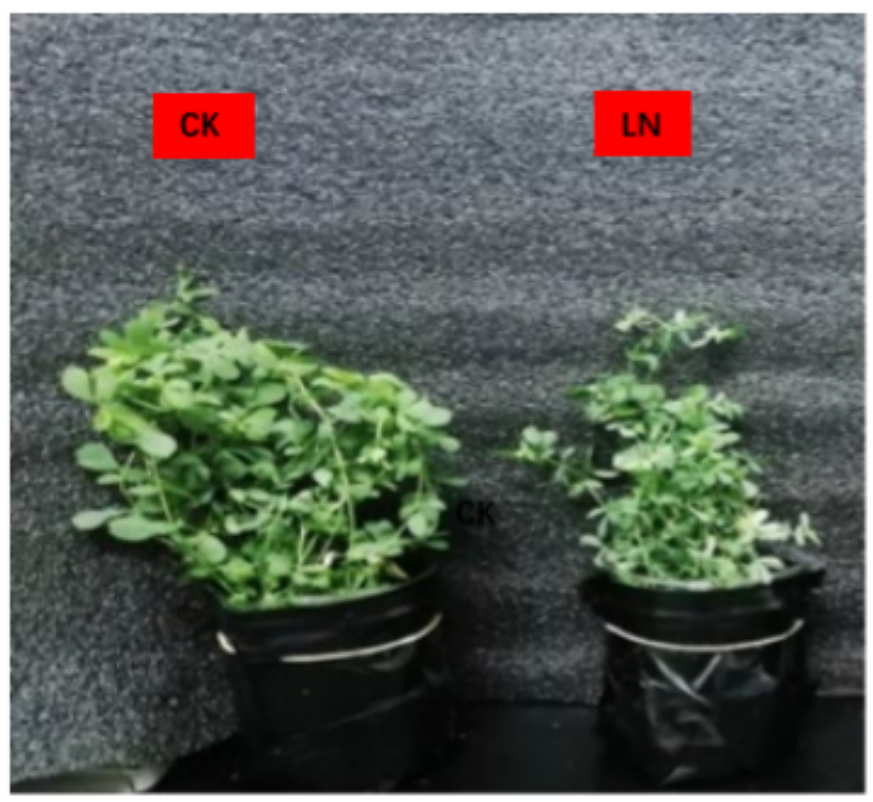

C

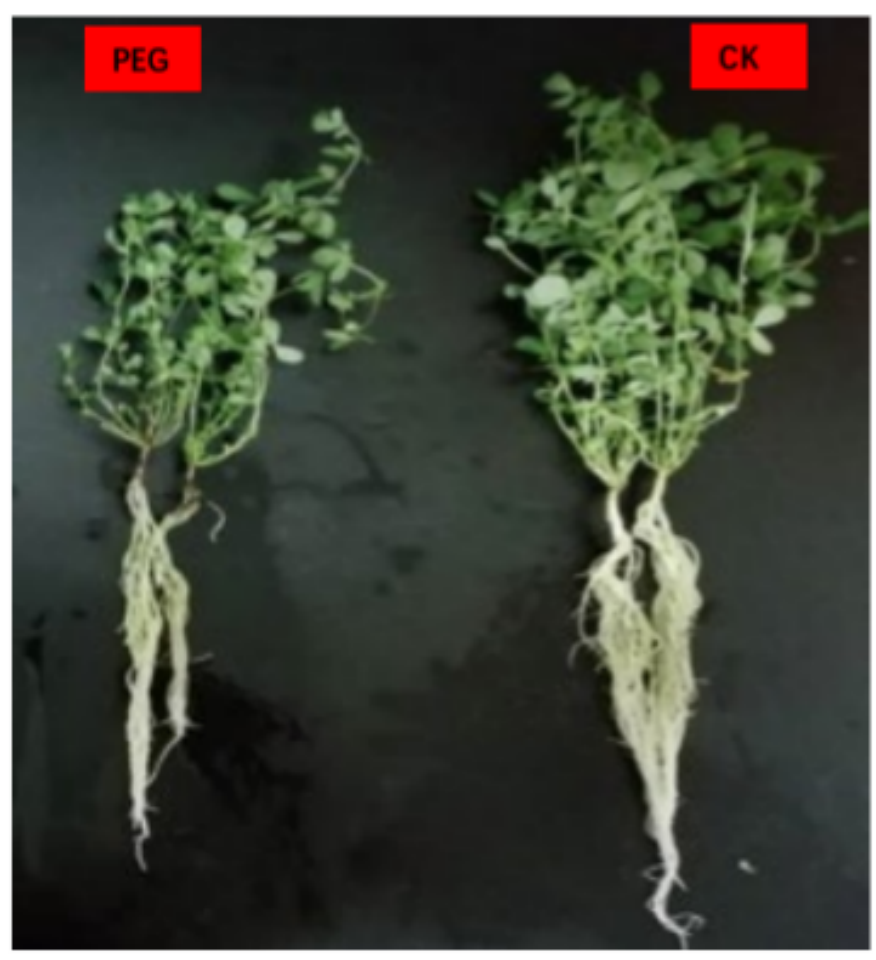

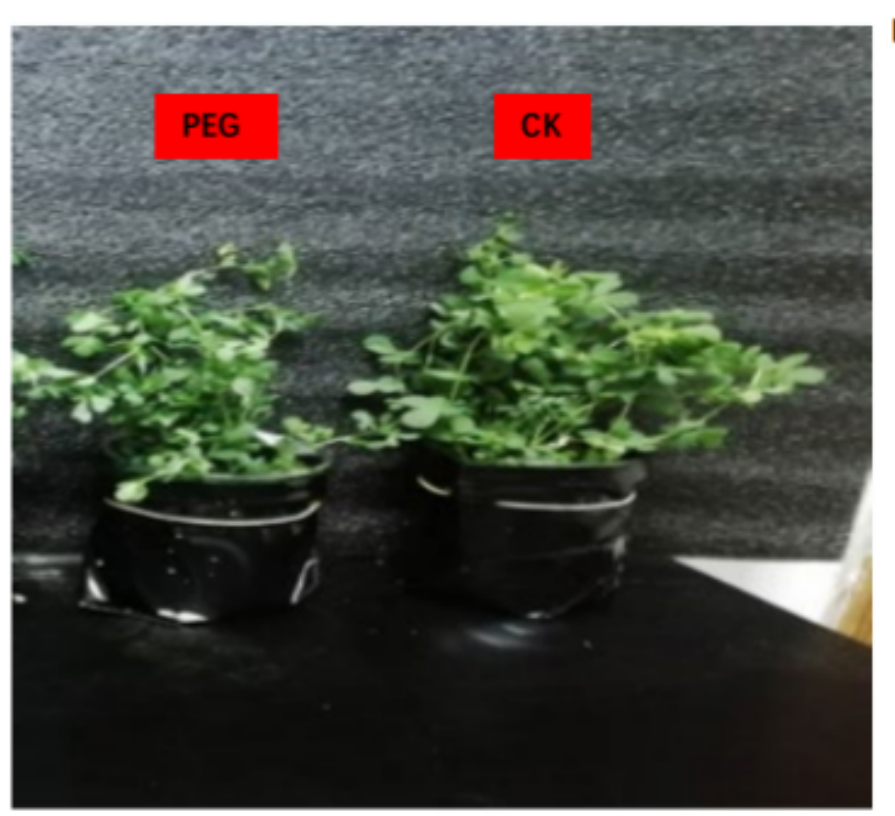

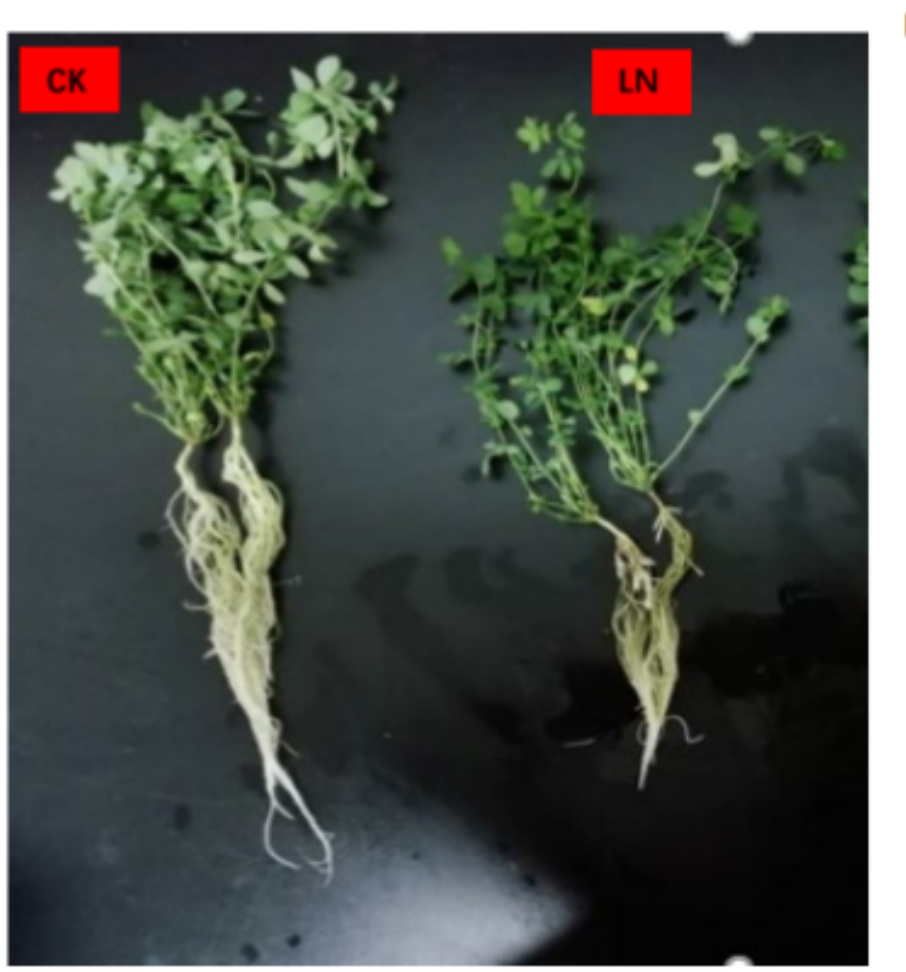

Figure 1 
Appearance characteristics of Lotus japonicus under low-phosphorus and drought stresses
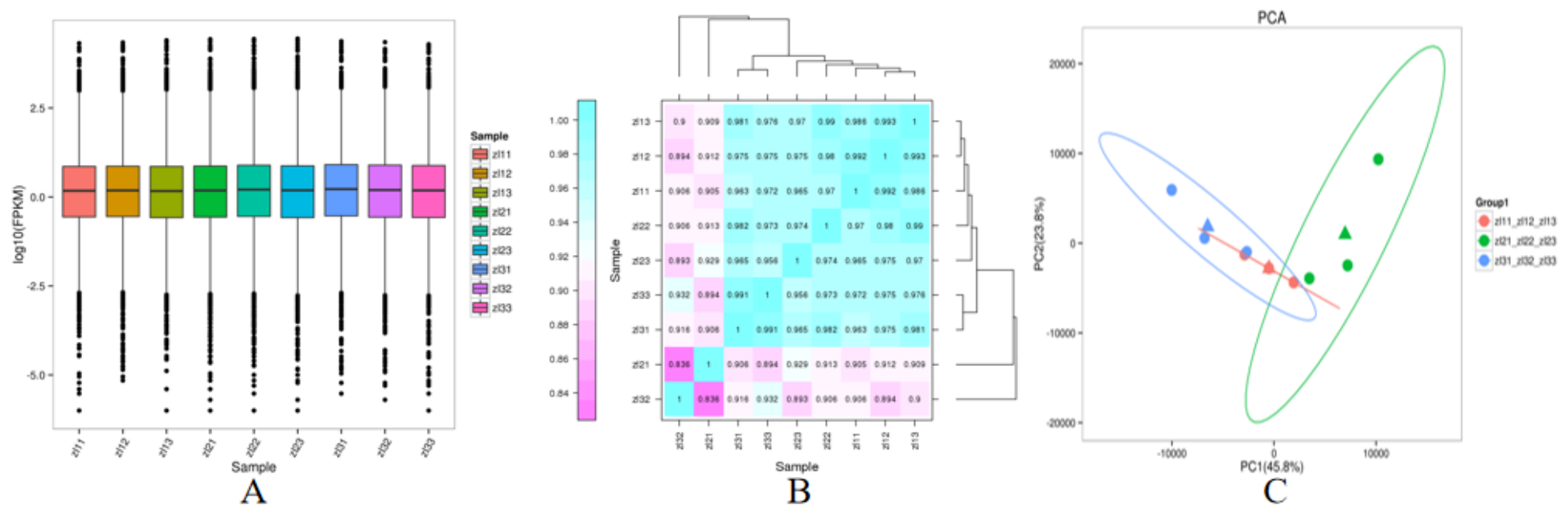

Figure 2

Gene expression analysis of samples. A: Box plot of the gene expression distribution of all samples. B: Heat map of expression level correlations among different products. C: PCA of gene expression in all samples, where the triangles represent the mean PCA results of the samples.

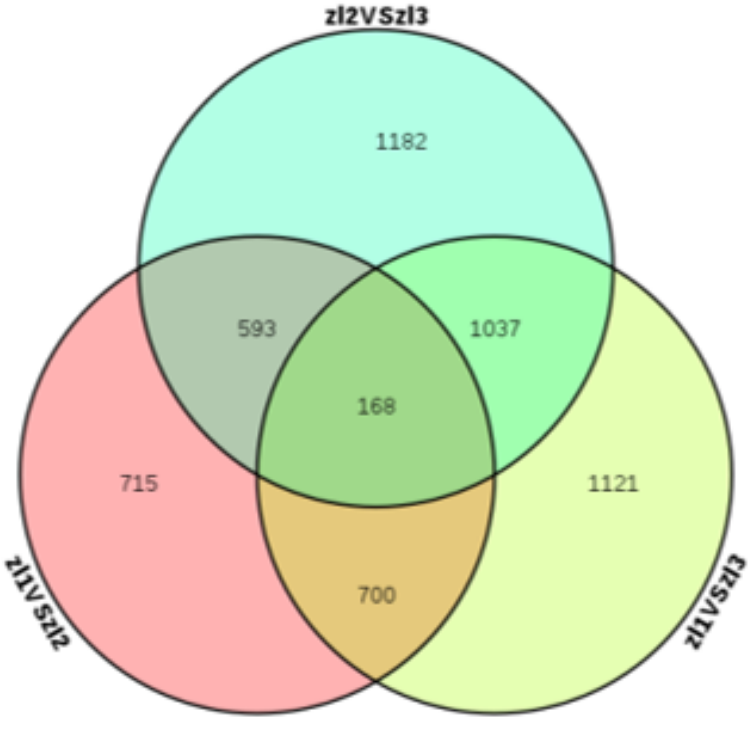

A

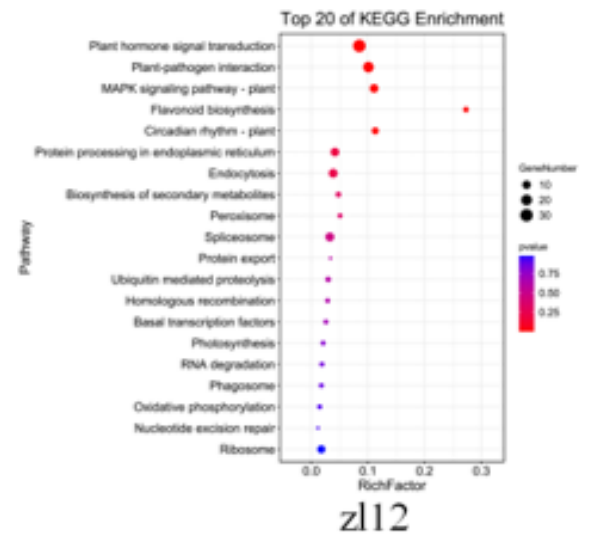

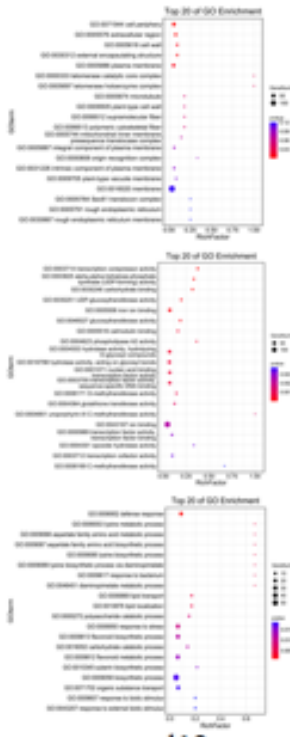

zl12

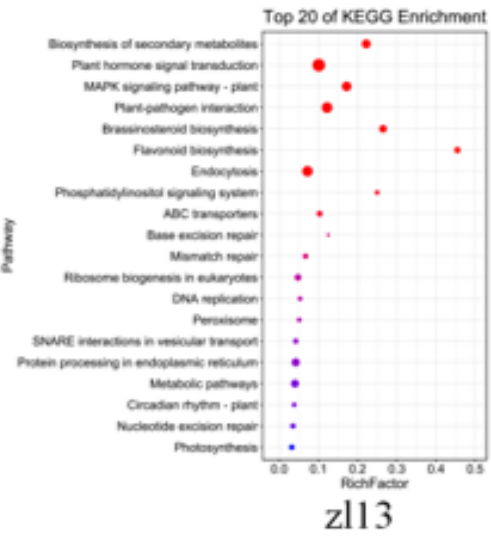

$\mathrm{C}$

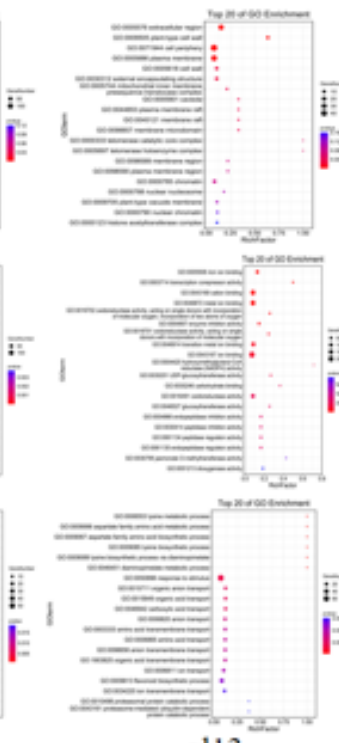

zl13

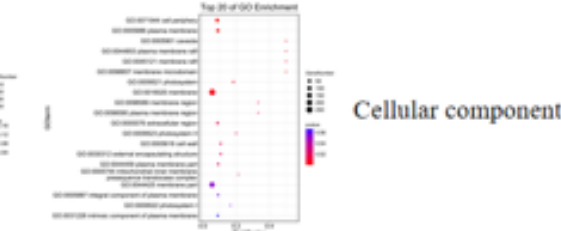

$\mathrm{B}$

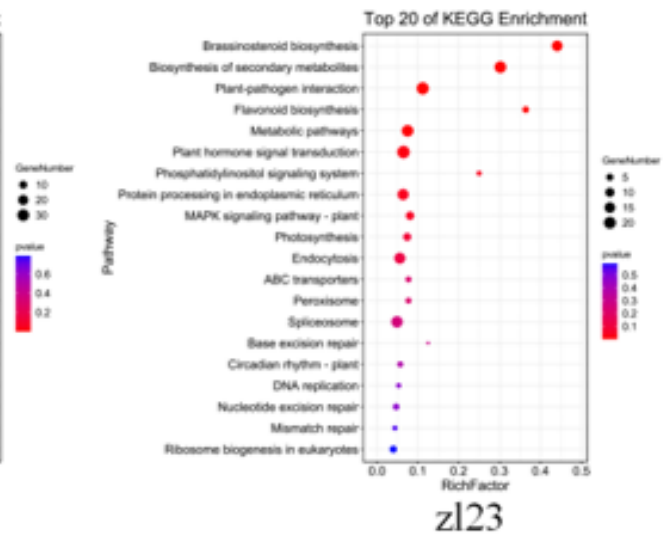

Page 23/29 


\section{Figure 3}

Analysis of differentially expressed genes. A: Venn diagram of differentially expressed genes. B: GO enrichment analysis showing the top 20 significantly enriched items. Each line from top to bottom represents cellular component, molecular function and biological processes. C: KEGG metabolic pathway analysis showing the top 20 significantly enriched pathways.

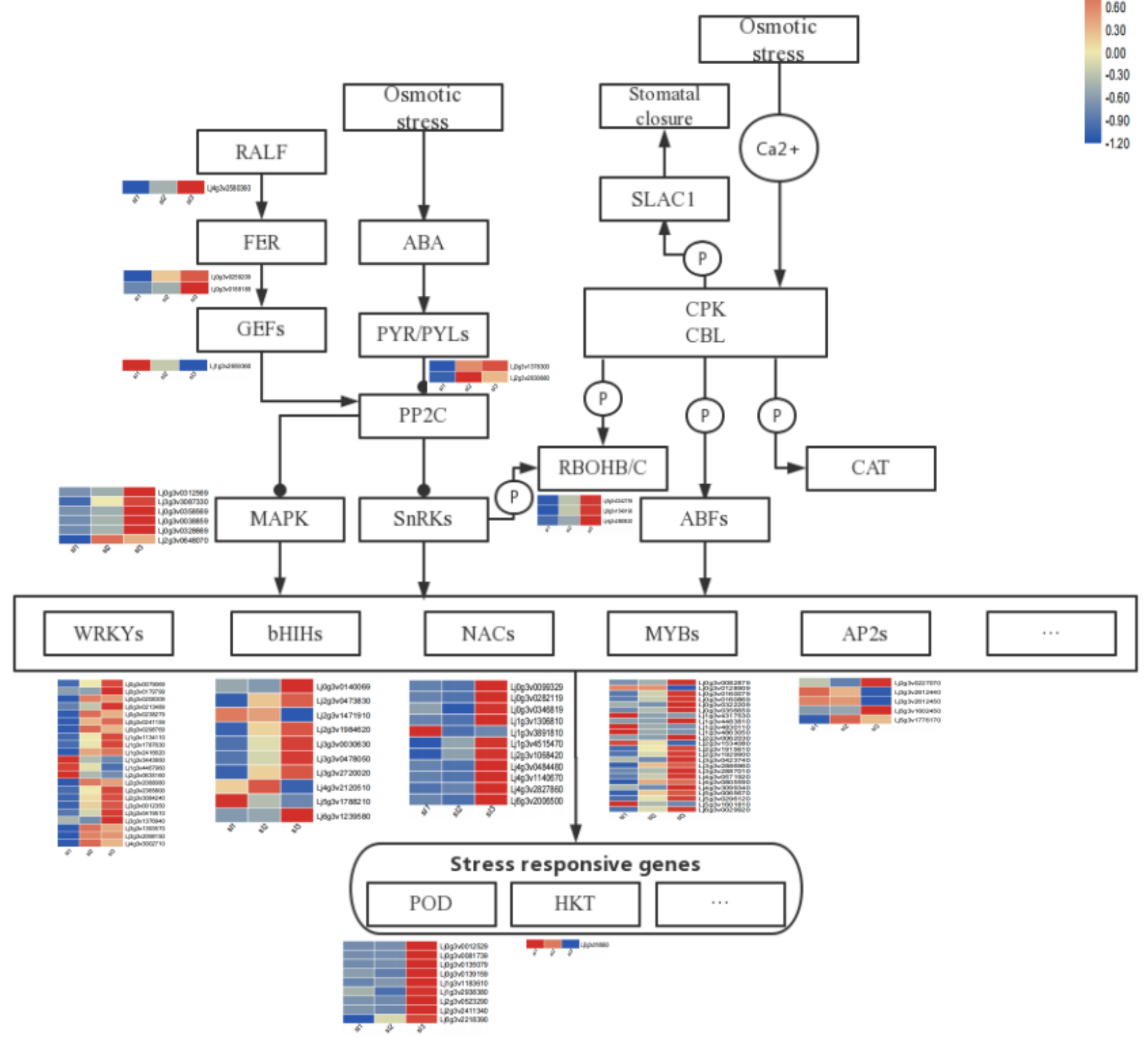

Figure 4

Flow chart of differentially expressed genes involved in the osmotic stress response under drought stress. The average FPKM values of the repeated genes in three samples from each treatment were uniformly 
selected, and the heat map was drawn by row normalization.

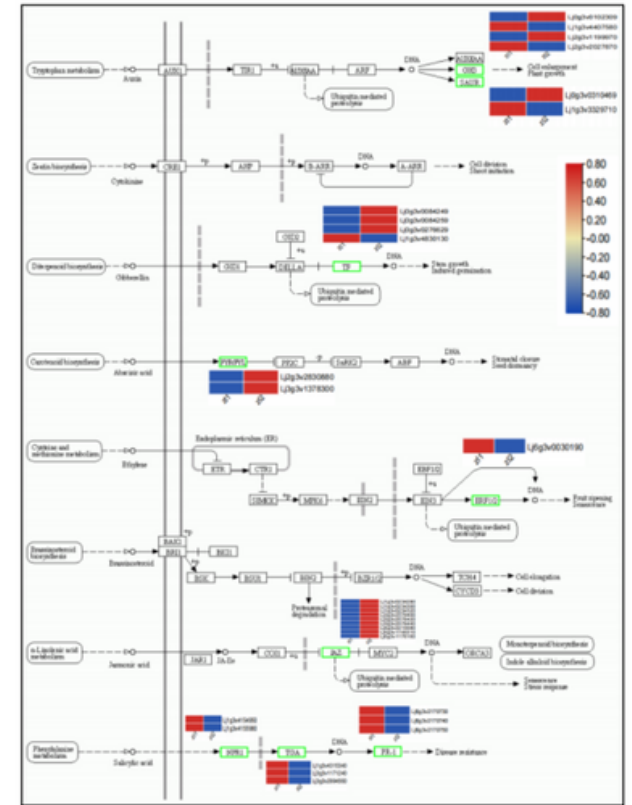

A

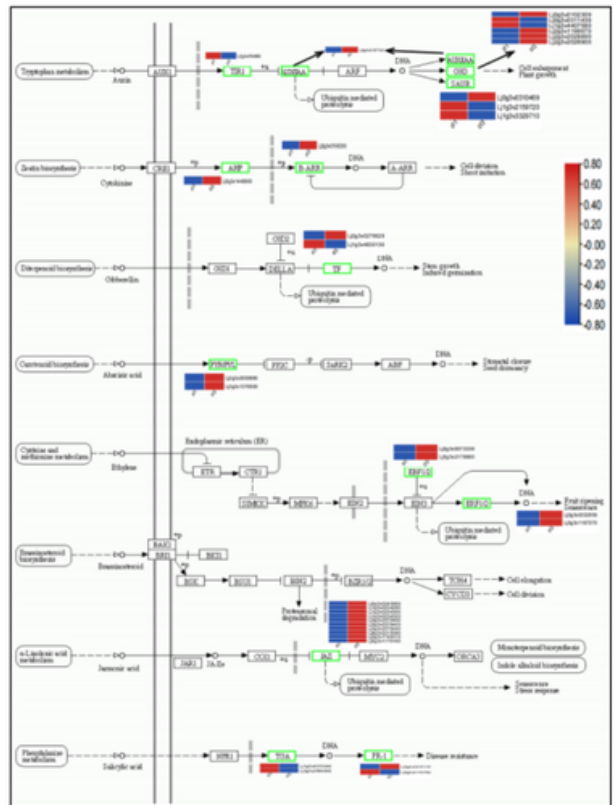

B

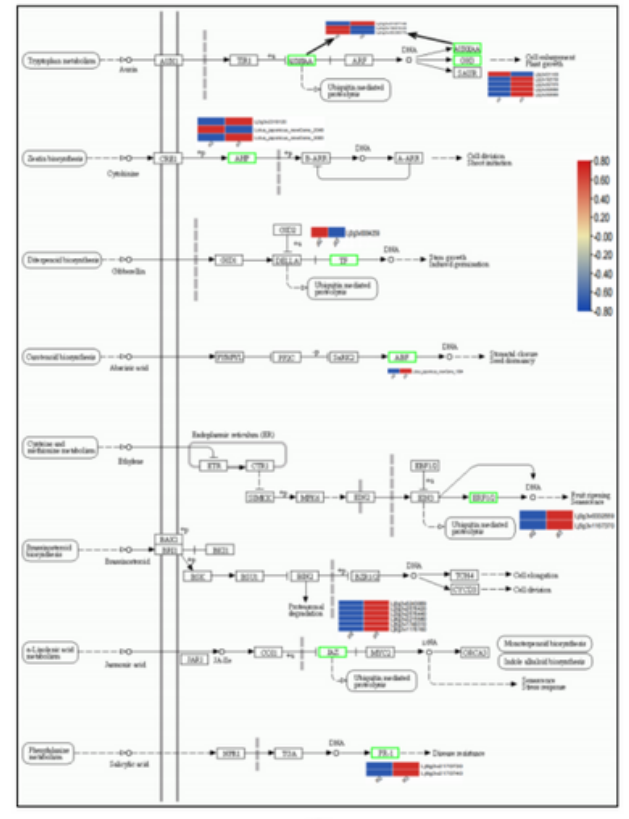

$\mathrm{C}$

\section{Figure 5}

Plant hormone signal transduction response genes. A: zl1 vs. zl2(z|12). B:zl1 vs. z|3(z|13). C: zl2 vs. $z \mid 3(z \mid 23)$. The average FPKM values of repeated genes in each sample under three replicates of each treatment were uniformly selected, and row normalization was used to draw heat maps. 

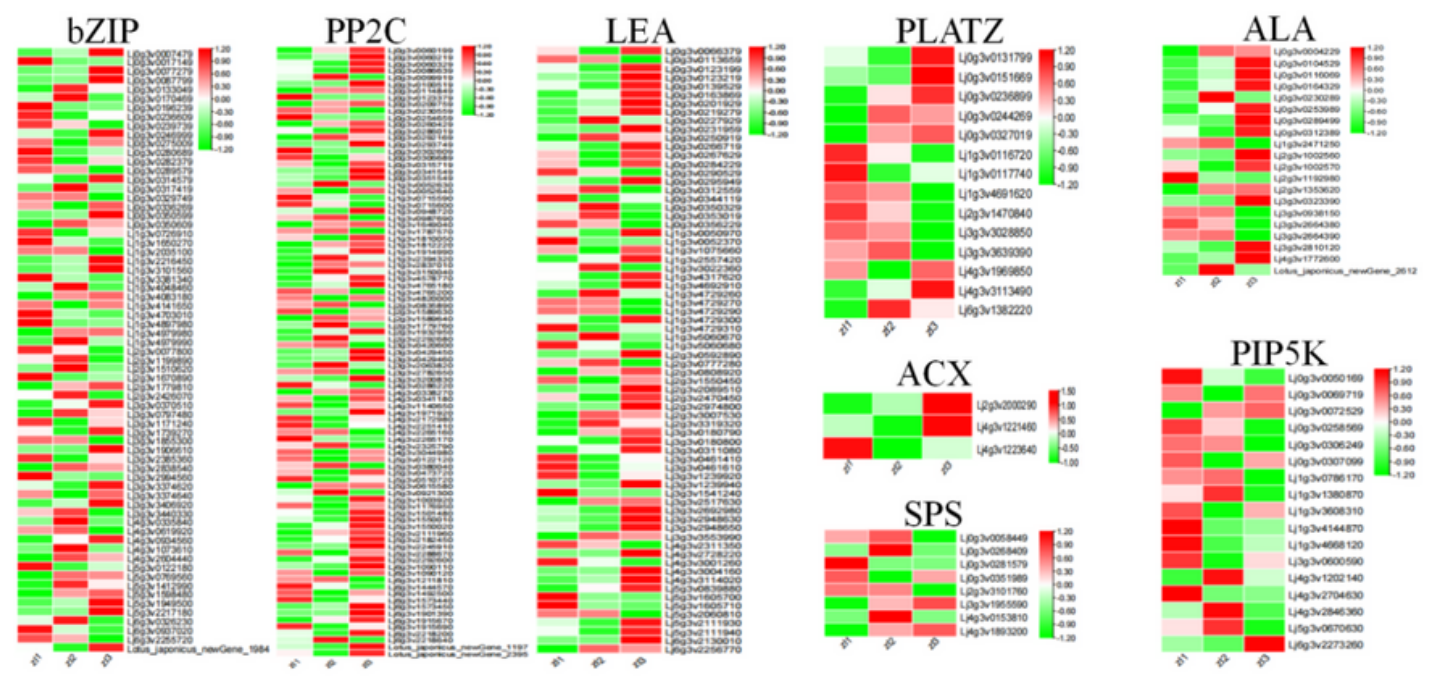

Figure 6

Thermograms of the expression of different transcription factor members under control, low-phosphorus and drought stresses. The average FPKM values of the repeated genes of each sample under three replicates of each treatment were uniformly selected, and row normalization was used to draw heat maps. 

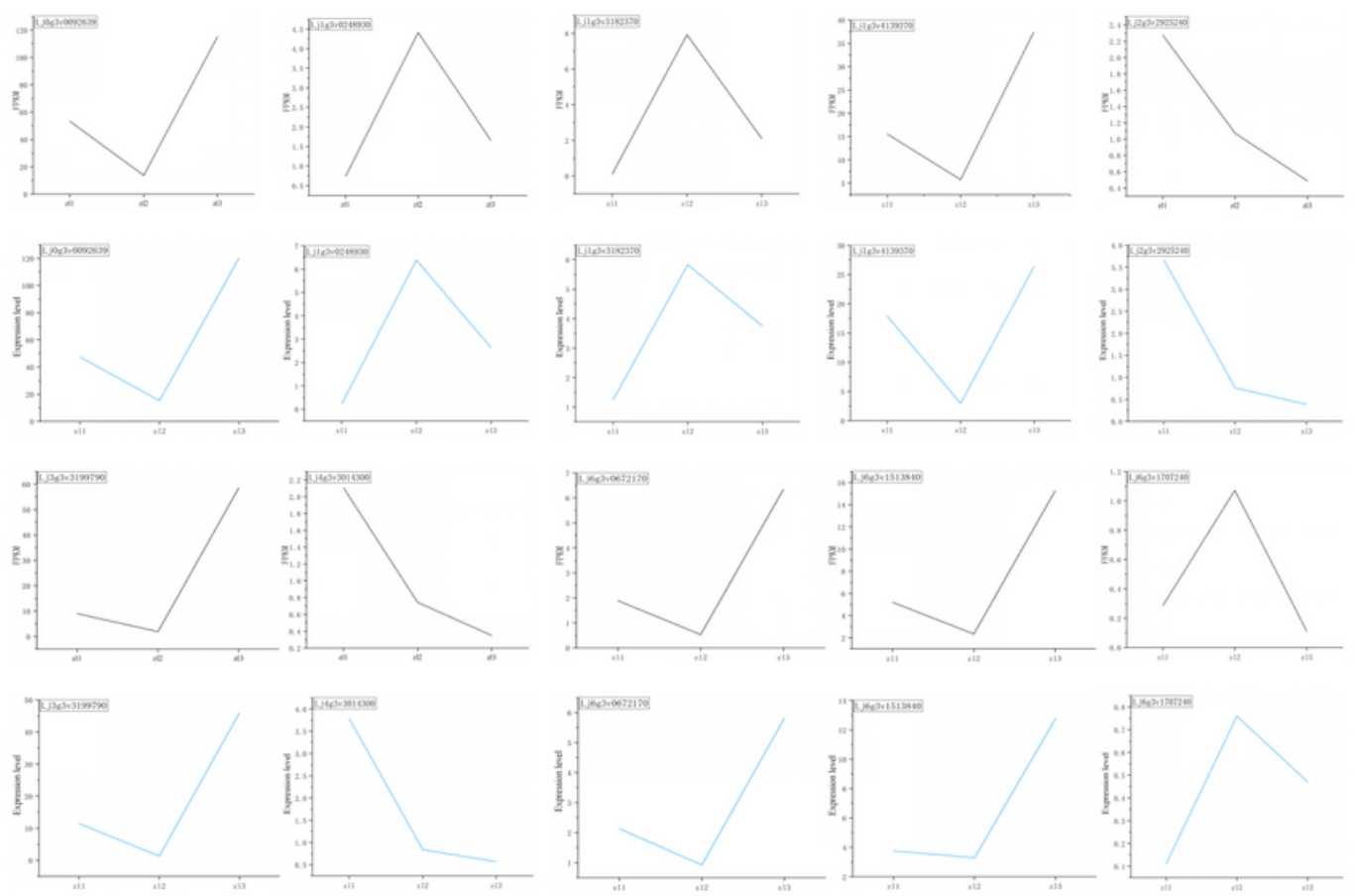

\section{Figure 7}

Quantitative comparison of gene expression and fluorescence. The black lines represent the gene FPKM values, and the blue lines represent the quantitative fluorescence values. The average FPKM values of the repeated genes of each sample under three replicates of each treatment were uniformly selected for mapping.
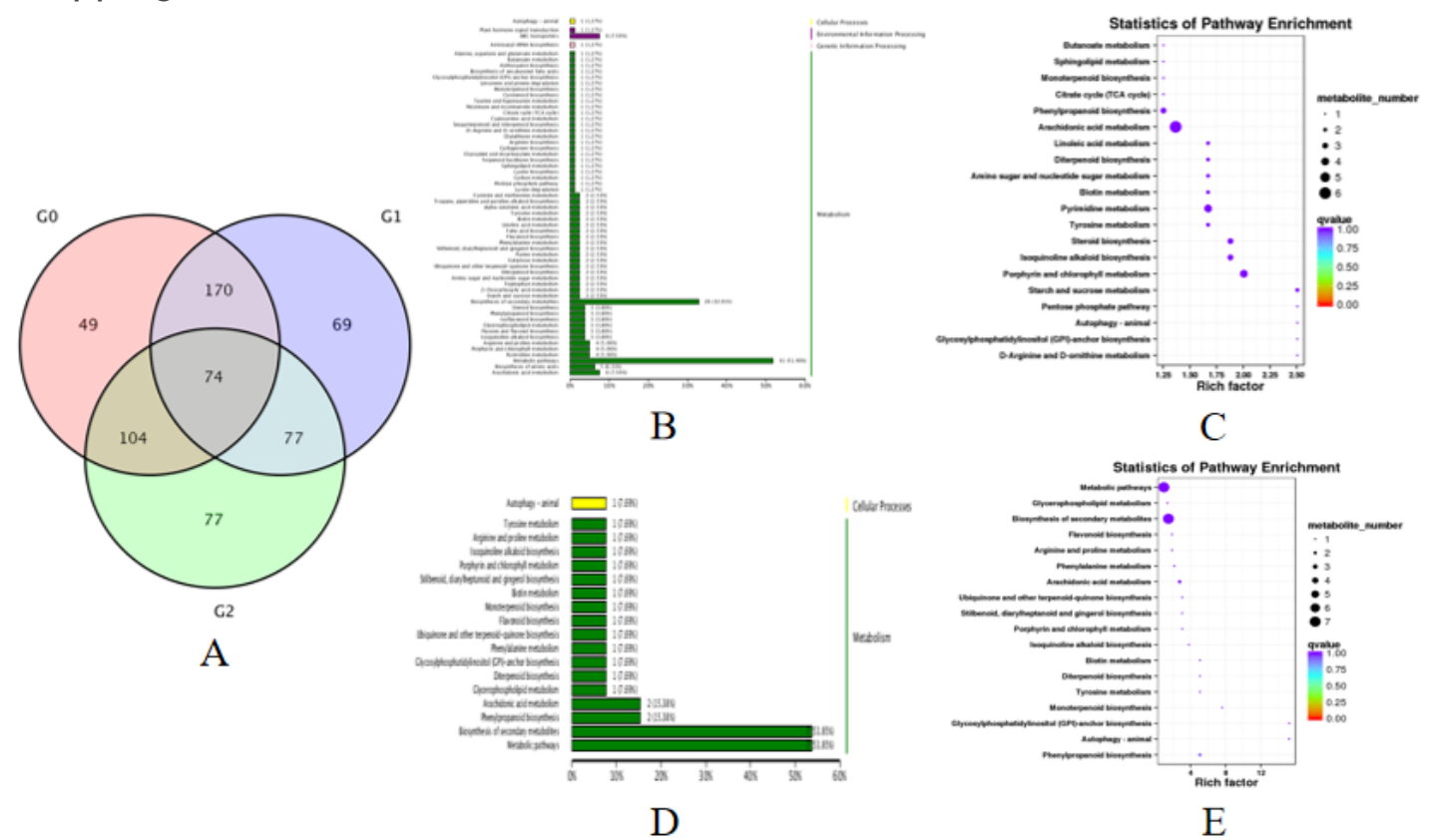

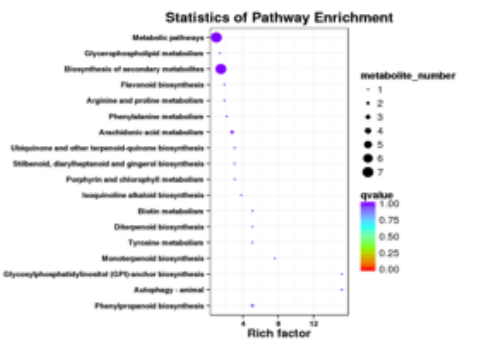

$\mathrm{E}$

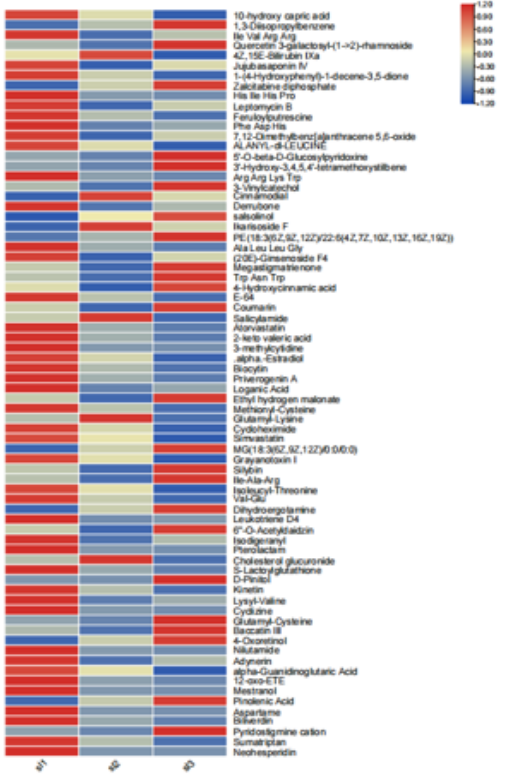

$\mathrm{F}$

Figure 8 
Differential metabolite analysis. G0: z|13, G1: z|12, G2: z|23. A: Venn diagram of differential metabolites between different treatments. B: KEGG pathway classification map of all differential metabolites. C: The KEGG enrichment of differential metabolites was significant in 20 pathways. D: KEGG pathway classification map of 74 common differential metabolites. E: The KEGG enrichment of 74 common metabolites was significant in 18 pathways. F: Quantitative heat map of 74 differential metabolites. The average value of each metabolite was calculated, and the row normalization method was used for analysis.

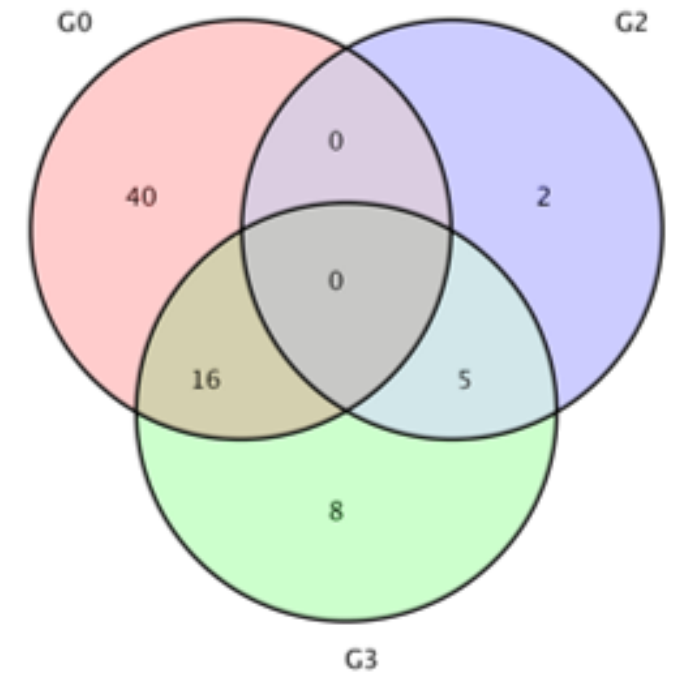

A

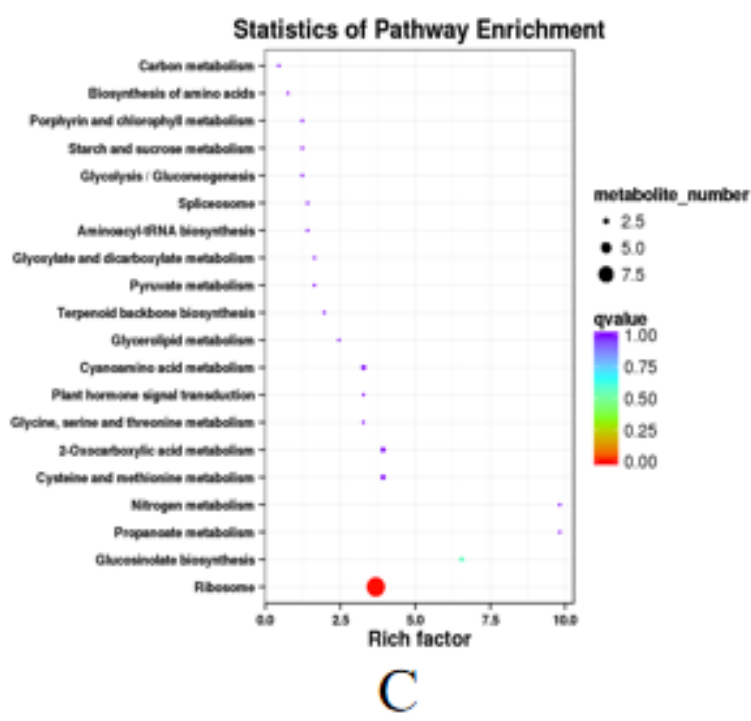

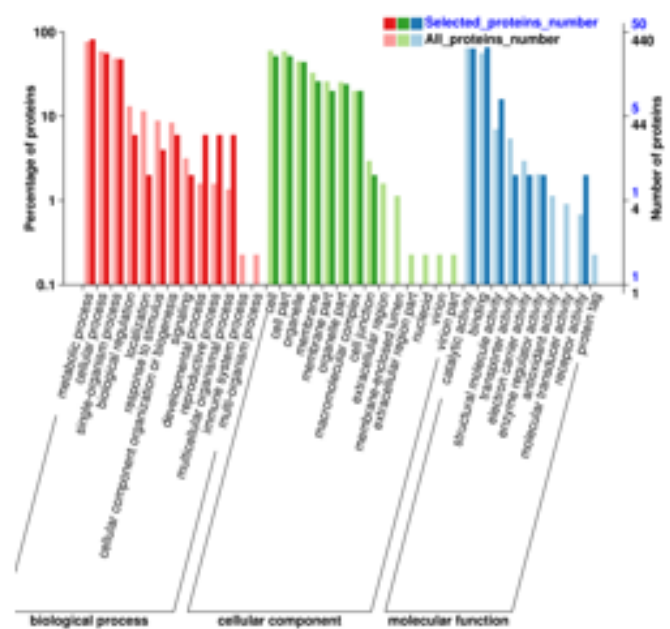

B

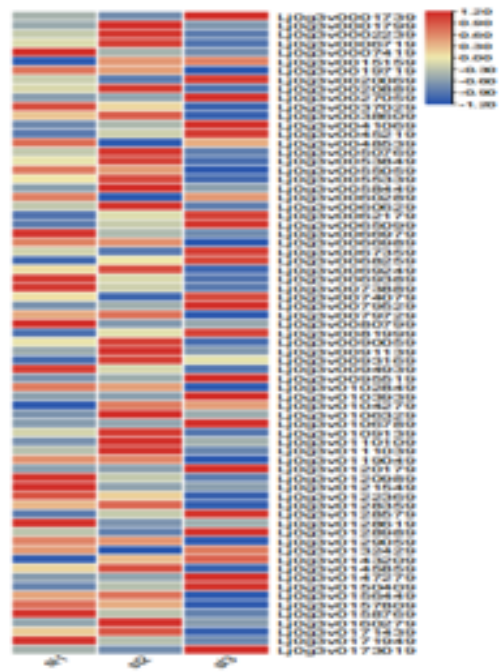

$\mathrm{D}$

Figure 9

Differential protein analysis. G0: sl12, G1: sl23, G2: sl13. A: Venn diagram of differentially expressed proteins. B: GO enrichment analysis of differentially expressed proteins. C: The top 20 pathways with significant KEGG enrichment of differentially expressed proteins. D: Quantitative heat map of 71 differentially expressed proteins. The row normalization method was used to analyze.

\section{Supplementary Files}


This is a list of supplementary files associated with this preprint. Click to download.

- Supplementarydata.zip 